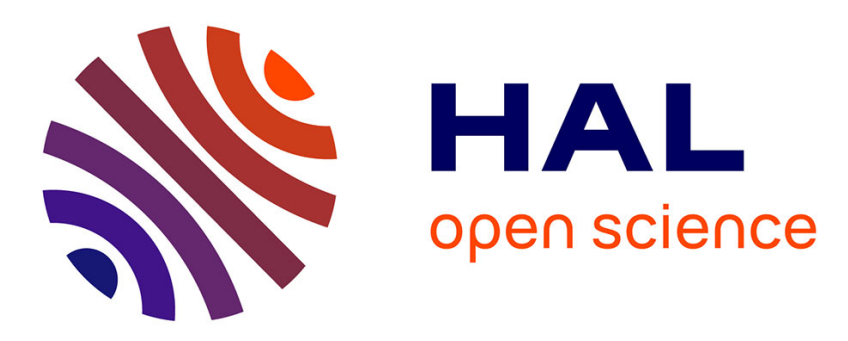

\title{
The Betti map associated to a section of an abelian scheme
}

Y. André, P. Corvaja, U. Zannier

\section{To cite this version:}

Y. André, P. Corvaja, U. Zannier. The Betti map associated to a section of an abelian scheme. Inventiones Mathematicae, 2020, 10.1007/s00222-020-00963-w . hal-02869609

\section{HAL Id: hal-02869609 \\ https://hal.sorbonne-universite.fr/hal-02869609}

Submitted on 16 Jun 2020

HAL is a multi-disciplinary open access archive for the deposit and dissemination of scientific research documents, whether they are published or not. The documents may come from teaching and research institutions in France or abroad, or from public or private research centers.
L'archive ouverte pluridisciplinaire HAL, est destinée au dépôt et à la diffusion de documents scientifiques de niveau recherche, publiés ou non, émanant des établissements d'enseignement et de recherche français ou étrangers, des laboratoires publics ou privés. 


\title{
THE BETTI MAP ASSOCIATED TO A SECTION OF AN ABELIAN SCHEME
}

\author{
Y. ANDRÉ, P. CORVAJA, U. ZANNIER
}

(WITH AN APPENDIX BY Z. GAO)

\begin{abstract}
Given a point $\xi$ on a complex abelian variety $A$, its abelian logarithm can be expressed as a linear combination of the periods of $A$ with real coefficients, the Betti coordinates of $\xi$. When $(A, \xi)$ varies in an algebraic family, these coordinates define a system of multivalued real-analytic functions. Computing its rank (in the sense of differential geometry) becomes important when one is interested about how often $\xi$ takes a torsion value (for instance, Manin's theorem of the kernel implies that this coordinate system is constant in a family without fixed part only when $\xi$ is a torsion section).

We compute this rank in terms of the rank of a certain contracted form of the KodairaSpencer map associated to $(A, \xi)$ (assuming $A$ without fixed part, and $\mathbb{Z} \xi$ Zariski-dense in $A$ ), and deduce some explicit lower bounds in special situations. For instance, we determine this rank in relative dimension $\leq 3$, and study in detail the case of jacobians of families of hyperelliptic curves.

Our main application, obtained in collaboration with Z. Gao, states that if $A \rightarrow S$ is a principally polarized abelian scheme of relative dimension $g$ which has no non-trivial endomorphism (on any finite covering), and if the image of $S$ in the moduli space $\mathcal{A}_{g}$ has dimension at least $g$, then the Betti map of any non-torsion section $\xi$ is generically a submersion, so that $\xi^{-1} A_{\text {tors }}$ is dense in $S(\mathbb{C})$.
\end{abstract}

\section{CONTENTS}

1. Introduction I: motivation.

2. Introduction II: main results.

3. The Betti map.

4. From Betti to Kodaira-Spencer.

5. Generic rank of the Betti map. 12

6. Abelian schemes with $\operatorname{End}_{S} A=\mathbb{Z}$ and $d_{\mu_{A}} \geq g$. Main theorem. 14

7. Case study I: $g \leq 3$. 16

8. Case study II: complex hyperelliptic case. 18

9. Case study III: real hyperelliptic case. 19

10. Appendix by Z. Gao: an application of the pure Ax-Schanuel Theorem. 23

References

Date: January 20, 2020.

1991 Mathematics Subject Classification. 11G, 11J, 14K, 14M.

Key words and phrases. Abelian scheme, torsion values, Zilber-Pink conjecture, Betti map, Kodaira-Spencer map, hyperelliptic curve, hyperbolic Ax-Schanuel theorem. 


\section{INTRODUCTION I: MOTIVATION.}

1.1. Let $A \rightarrow S$ be an abelian scheme of relative dimension $g$ over a (non necessarily complete) complex algebraic variety, and let $\xi: S \rightarrow A$ be a section. We assume that $\xi$ is not identically torsion, i.e. does not take value in the kernel $A[n]$ of multiplication by any positive integer $n$ in $A$. A general problem which arises in a number of contexts concerns the distribution of torsion values of $\xi$, or more accurately, of the points $s \in S$ such that $\xi(s)$ is a torsion point on the abelian variety $A_{s}$.

Let us introduce the closed subschemes of $S$ :

$$
\xi^{-1} A[n]=\{s \in S: n \xi(s)=0\}, \quad n=1,2, \ldots
$$

Since $A[n]$ is finite etale over $S$, it is the disjoint union of closed subschemes corresponding to exact $m$-torsion for $m \mid n$, and $\xi^{-1} A[n]$ decomposes accordingly. Each non-empty component has codimension $\leq g$ in $S$, in virtue of Serre's codimension theorem [33, Th. 3, V-18]. In the general framework of Pink's conjectures ${ }^{1}$, the following heuristic dichotomy then arises:

(A) When $\operatorname{dim} S<g$, one expects under natural assumptions that $\xi^{-1} A_{\text {tors }}$ is contained in $\bigcup_{n=1}^{N} \xi^{-1} A[n]$ for some $N$, hence is not Zariski-dense. This is a typical question in the theory of "unlikely intersections", falling into the framework of the Pink-Zilber conjecture (see [7]).

(B) When $\operatorname{dim} S \geq g$, one expects on the contrary, under natural assumptions to be discussed in the sequel, that $\xi^{-1} A_{\text {tors }}$ is dense, even in the complex topology. This will be the guiding thread of this work.

1.2. A useful tool to investigate the distribution of torsion values is provided by the socalled Betti map: any complex abelian variety $A$ of dimension $g$ may be presented as a complex torus $\mathbb{C}^{g} / \mathcal{L} \cong\left(\mathcal{L} \otimes_{\mathbb{Z}} \mathbb{R}\right) / \mathcal{L}$, and every point $\xi$ of $A$ may then be identified by its $2 g$ real coordinates in a mesh of the lattice $\mathcal{L}$. The aim of this paper is to understand how these so-called "Betti coordinates" vary when $(A, \xi)$ moves in an algebraic family; in fact, one gets in this way a multivalued real-analytic map $\beta$, the Betti map, from the parameter space $S$ to $\mathbb{R}^{2 g}$.

While this problem could have been raised in the XIXth century, we are not aware of any trace until recent occurrences in algebraic and diophantine geometry, where the relevance of the Betti map arises from the fact that its rational values correspond to torsion values of the section $\xi$.

1.3. Here is a sample of classical or recent occurrences of torsion value problems and/or Betti maps.

(i) What may be the first explicit connection between torsion value problems and Betti maps can be found in a celebrated paper by Yu. Manin [23]. He connected the map with differential operators, leading to what is nowadays, after Grothendieck, called the GaussManin connection (for families of curves). A byproduct of his analysis was a characterization of the cases when the Betti map is locally constant. If the abelian family has no fixed part, he proved that the Betti coordinates are locally constant if and only if these constants are rational, i.e. if and only if the section is identically torsion ${ }^{2}$.

(ii) Torsion values occur in connection to Poncelet's game and its higher dimensional generalizations in a paper by Ph. Griffiths and J. Harris [15]: given two conics, construct a

\footnotetext{
${ }^{1}$ formulated in [30] and further discussed in [31]; see also the third author's book [38], especially chapter 3).

${ }^{2}$ for the convoluted story of the proof of this theorem, see for instance $[5][3, \S 1]$.
} 
polygon which is inscribed in one conic and circumscribed to the other one. Jacobi proved that the pair of conics determines an elliptic curve with a given point, such that such a polygon can be constructed if and only if the given point is torsion. Following Jacobi, Griffiths and Harris took a "variational approach" to this game, by varying the two conics, so obtaining a section of an elliptic family where the base parametrizes the pairs of conics; again torsion comes into play, being connected to the existence of such a polygon (and to the finiteness of the game). They also considered a three-dimensional situation, where conics are replaced by quadric surfaces and polygons by polyhedra. Again, the Betti map appears linking the behaviour of the game with variations of the quadrics. In this context, the density of the torsion values in the base is far from obvious, and they prove it only in certain hypersurfaces of the base. More recently, the issue of torsion in the context of Poncelet's theorem has been revisited by N. Hitchin, and turned out to be related to the Painlevé VI equation [17] (see also work in progress by G. Wüstholz).

(iii) Torsion values and the Betti map also occur in the context of Pell equations in polynomials, which were already studied by Abel in connection with integration of differentials; motivated by this context, B. Mazur recently raised an issue concerning the "Pellian" hyperelliptic curves in the space of all hyperelliptic curves of given genus (see [7]).

(iv) These Pell equations in polynomials have number theoretic significance, but also appear in investigations related to the spectral theory of periodic discrete Schrödinger equations (see [19]).

(v) The Pell equation involving the hyperelliptic family of given genus, but now restricting to field of real numbers, appears also in an issue raised by J.-P. Serre, in connection with an incomplete argument in a classical paper by R. Robinson [32]. An argument for filling this gap was given by B. Lawrence, and we offer an independent one in section 9.

(vi) Still from a different perspective, C. Voisin [36] considered very recently a closely related problem, this time motivated by the investigation of Chow groups. In the context of Lagrangian fibrations on hyperkähler manifolds, she uses methods of her own for the case $g \leq 2$, and our Corollary 2.2.4 to prove the desired conclusion for $g \leq 4$.

(vii) Betti coordinates also occur in a forthcoming work by Z. Gao and Ph. Habegger on the geometric Bogomolov conjecture [12].

1.4. In this paper, we undertake a systematic study of the Betti map $\beta$ associated to $(A, \xi)$. We relate the derivative of the Betti map to the Kodaira-Spencer map; under a suitable condition on the abelian scheme, expressed in terms of the Kodaira-Spencer map, we prove that the Betti map of every section not contained in an algebraic subgroup is a submersion; in the case of an abelian scheme whose generic fibre has no non-trivial endomorphisms, our result is essentially best possible (see Theorem 2.3.1).

We then deduce the density of torsion values, for every section, under some fairly natural hypotheses.

Functional transcendence also appears, through a monodromy theorem by the first author, and also via a very recent result, namely the proof by N. Mok, J. Pila, J. Tsimerman [25] of a version of Ax-Schanuel conjecture in the context of subvarieties of the moduli space $\mathcal{A}_{g}$ and its uniformization.

\section{INTRODUCTION II: MAIN RESULTS.}

2.1. Rank of the Betti map and torsion values. Let $A \rightarrow S$ be an abelian scheme of relative dimension $g$ over a smooth complex algebraic variety of dimension $d$, and let $\xi$ : 
$S \rightarrow A$ be a section. Let $\beta: \tilde{S} \rightarrow \mathbb{R}^{2 g}$ be the associated (real analytic) Betti map, where $\tilde{S}$ denotes the universal covering of $S(\mathbb{C})(c f . \S 3$ for a precise definition and discussion of this map) $)^{3}$. In this paper, we focus on the (generic) rank of $\beta$ as defined in differential topology, namely, the maximal value of the rank of the derivative $d \beta(\tilde{s})$ when $\tilde{s}$ runs through $\tilde{S}$. We denote it by $\operatorname{rk} \beta$.

As explained above, the motivation for studying $\operatorname{rk} \beta$ comes from the relation to torsion values of $\xi$. The following proposition, which is a simple consequence of the constant rank theorem and of the fact that $\mathbb{Z}[1 / p]$ is dense in $\mathbb{R}$, makes this relation explicit.

2.1.1. Proposition. The following are equivalent:

(1) $\operatorname{rk} \beta \geq 2 g$,

(2) $\beta$ is submersive on a dense open subset of $\tilde{S}$,

(3) the image of $\beta$ contains a non-empty open subset of $\mathbb{R}^{2 g}$, and imply ${ }^{4}$ :

(4) for every prime $p, \xi^{-1} A\left[p^{\infty}\right]$ is dense in $S(\mathbb{C})$ (for the complex topology),

(5) $\xi^{-1} A_{\text {tors }}$ is dense in $S(\mathbb{C})$.

Because $\mathrm{rk} \beta$ is invariant by dominant pull-back $S^{\prime} \rightarrow S$, one may assume that for fixed $n \geq 3, A[n]$ is a disjoint union of copies of $S$ (i.e. $n$-torsion is $S$-rational). One may also replace $A$ by any isogenous abelian scheme and $\xi$ by the corresponding pull-back, and assume in particular that $A$ is principally polarized.

This gives then rise to a modular map $\mu_{A}: S \rightarrow \mathcal{A}_{g, n}$ such that $A$ is the pull-back by $\mu_{A}$ of the universal principally polarized abelian scheme (with level $n$ structure) $\mathcal{X}_{g, n}$ on $\mathcal{A}_{g, n}$. Moreover $\xi$ gives rise to a modular map $\mu_{\xi}: S \rightarrow \mathcal{X}_{g, n}$ lifting $\mu_{A}$

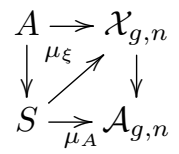

such that $\xi$ is the pull-back by $\mu_{\xi}$ of the diagonal map $\mathcal{X}_{g, n} \rightarrow \mathcal{X}_{g, n} \times_{\mathcal{A}_{g, n}} \mathcal{X}_{g, n}$.

If $d_{\mu_{\xi}}$ denotes the dimension of the image of $\mu_{\xi}$, we have the upper bound

$$
\operatorname{rk} \beta \leq 2 \cdot \min \left(d_{\mu_{\xi}}, g\right) \text {. }
$$

A plausible guess is that if $\mathbb{Z} \xi$ is Zariski-dense in $A$, and $A$ has no fixed part (over any finite etale covering of $S$ ), (2.1) is an equality ${ }^{5}$.

2.2. Formulas for $\mathrm{rk} \beta$. Passing to universal coverings, $\mu_{A}$ lifts to a map

$$
\tilde{\mu}_{A}: \tilde{S} \rightarrow \mathfrak{H}_{g}, \quad \tilde{s} \mapsto Z(\tilde{s}),
$$

toward the Siegel space, where $Z=\Omega_{2} \Omega_{1}^{-1}$ is given in terms of abelian periods as usual. Let also $L: \tilde{S} \rightarrow \mathbb{C}^{g}$ stand for an abelian logarithm of the section $\xi: S \rightarrow A$ multiplied on the right by $\Omega_{1}^{-1}(c f .4 .1)$.

Our first formula is analytic:

\footnotetext{
${ }^{3}$ actually, it also depends on a choice of branch of abelian logarithm $\lambda, c f .3 .1$.

4 we do not know whether the converse holds: the image of $\beta$ might be dense without containing a dense open subset.

5 as Z. Gao pointed out [13], this guess should be slightly amended to take into account the fact that the right hand side of (2.1) is not always additive in $A / S$.
} 
2.2.1. Theorem. Assume that $A$ has no fixed part (over any finite etale covering of $S$ ), and $\mathbb{Z} \xi$ is Zariski-dense in $A$. Up to replacing $S$ by an affine dense open subset, we may assume that its tangent bundle is free, generated by d derivations $\partial_{1}, \ldots, \partial_{d}$. Then

$$
\operatorname{rk} \beta=2 \cdot \max _{\mu_{0}, \ldots, \mu_{g} \in \mathbb{C}^{g+1}} \max _{\tilde{s} \in \tilde{S}} \operatorname{rk}\left(\mu_{0} \partial_{i} L_{j}(\tilde{s})-\sum_{k=1}^{k=g} \mu_{k} \partial_{i} Z_{k j}(\tilde{s})\right)_{i j} .
$$

By letting $\mu_{0}=0$, one deduces the following criterion for generic submersivity, which does no longer involve the section:

2.2.2. Corollary. In the same situation, assume that $d \geq g$ and that $\operatorname{rk} \beta<2 g$. Then for any $\tilde{s} \in \tilde{S}$ and any $\mu \in \mathbb{C}^{g}$, there exists a complex-analytic subvariety of $\tilde{S}$ passing through $\tilde{s}$, of dimension $\geq d+1-g$, on which $Z . \mu$ is constant.

Our second formula is nothing but an algebraic intrinsic reformulation of the former in terms of Kodaira-Spencer maps ( $c f$. $\S 4.4)$. Recall the Kodaira-Spencer map of $A$ is the $\mathcal{O}_{S}$-linear map

$$
\theta_{A}: T_{S} \otimes \Omega_{A} \rightarrow \mathcal{H}_{\mathrm{dR}}^{1}(A / S) / \Omega_{A} \cong \text { Lie } A,
$$

induced by the Gauss-Manin connection. Note that the vector bundle $\Omega_{A}$ of invariant 1 -forms is the $g r^{1}$-notch of the Hodge filtration, whereas its dual Lie $A$ appears as the $g r^{0}$-notch via the polarization (see $\S 4.5$ for a presentation and discussion of $\theta_{A}$ ).

The Kodaira-Spencer map of the 1-motive $\left[\mathbb{Z}^{1 \mapsto \xi} \rightarrow A\right]$ attached to $(A, \xi)$ (in the sense of $[9, \mathrm{X}])$ is an enhanced version of $\theta_{A}$

$$
\theta_{\xi}: T_{S} \otimes g r^{1} \rightarrow g r^{0}
$$

where $g r^{1}=\Omega_{A}$ again, but $g r^{0}$ is an extension of Lie $A$ by $\mathcal{O}_{S}$ (cf. 4.5).

Assuming for simplicity that $S$ is affine, we denote by

$$
\theta_{\xi}^{\varpi}: T_{S} \rightarrow \text { Lie } A=\left(g r^{1}\right)^{\vee}
$$

the contracted map, for any section $\varpi$ of $\left(g r^{0}\right)^{\vee}$.

2.2.3. Theorem. Assume that A has no fixed part, and $\mathbb{Z} \xi$ is Zariski-dense in A. Then

$$
\operatorname{rk} \beta=2 \cdot \max _{\left(\varpi_{0}, \ldots, \varpi_{g}\right) \in\left(g r^{0}\right) \vee} \max _{s \in S} \operatorname{rk} \theta_{\xi}^{\varpi}(s) .
$$

By letting $\varpi_{0}=0$, one deduces the following criterion for generic submersivity in terms of $\theta_{A}$ (which again does not involve the section) ${ }^{6}$ :

2.2.4. Corollary. Under the same assumptions, if there exists $\omega \in \Gamma \Omega_{A}$ such that the map

$$
\theta_{A}^{\omega}: T_{S} \rightarrow \text { Lie } A, \partial \mapsto \theta_{A}(\partial \otimes \omega),
$$

has generic rank $g$, then $\mathrm{rk} \beta \geq 2 g$.

Let us give an indication on the method of proof of Theorem 2.2.1. For any $\nu \in \mathbb{C}^{2 g}$, let $I_{\nu}$ be the $d \times g$-matrix with entries $\partial_{i} \Lambda_{j}+\sum_{k} \nu_{k} \partial_{i} \Omega_{k j}$, where $\Lambda$ is a vector of abelian logarithms and $\Omega_{j k}$ a matrix of abelian periods (cf. (4.5) below). The rank of $d \beta(\tilde{s})$ can be computed in terms of vanishing/non vanishing of minors of $I_{-\beta(\tilde{s})}(c f .(4.7))$. The point is then to show that the vanishing of such a minor identically on $\tilde{S}$ implies the vanishing of the corresponding minor of $I_{\nu}$ for any $\nu$.

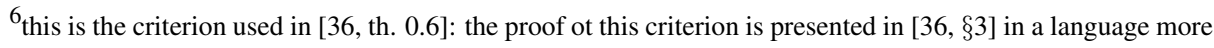
congenial to Hodge theoretists.
} 
The idea is to use monodromy via a theorem about relative monodromy of abelian logarithms with respect to abelian periods [2, Th. 3]. A straightforward application does not work directly because $I_{-\beta}$ is monodromy-invariant. The trick is that, after multiplication by some power of $\operatorname{det}\left(\begin{array}{cc}\Omega_{1} & \bar{\Omega}_{1} \\ \Omega_{2} & \bar{\Omega}_{2}\end{array}\right), I_{-\beta}$ becomes a polynomial in holomorphic and antiholomorphic multivalued functions on $S$, so that one can let two copies of $\pi_{1}(S)$ act on $\mathcal{O}(\tilde{S}) \otimes \overline{\mathcal{O}}(\tilde{S})$, instead of standard monodromy (which corresponds to the diagonal action).

2.3. Applications. Let $d_{\mu_{A}} \leq d$ be the dimension of the image of the modular map $\mu_{A}$. Our main application, in collaboration with Z. Gao, is:

2.3.1. Theorem. (with Z. Gao) Assume that the abelian scheme $A \rightarrow S$ has no non-trivial endomorphism over any finite covering of $S$, and that the section $\xi$ is non-torsion. Assume moreover that $d_{\mu_{A}} \geq g$. Then $\operatorname{rk} \beta \geq 2 g$, hence the set of points such that $\xi(s)$ is a torsion point is dense in $S(\mathbb{C})$.

(The first assumption means that the geometric generic fiber satisfies End $A_{\overline{\mathbb{C}(S)}}=\mathbb{Z}$ ). This theorem is obtained by combining Corollary 2.2.2, a classification of the abelian schemes under consideration ( $(6)$, and the recent theorem of Ax-Schanuel for $\mathcal{A}_{g}$ by Mok, Pila, Tsimerman (cf. App. 2). ${ }^{7}$

Besides, we study three situations where the rank of the Betti map can be proved to be maximal by independent and simpler means. In $\S 7$, Using Corollary 2.2.4, an elementary analysis of webs of quadrics, combined with a result of [4], allows to settle the case $g \leq 3$ :

2.3.2. Theorem. Suppose that the abelian scheme $A \rightarrow S$ has relative dimension $g \leq 3$, has no fixed part, and $d_{\mu_{A}} \geq g$. Then for every section $\xi$ not contained in a proper subgroup scheme, $\mathrm{rk} \beta \geq 2 g$.

In $\S 8$, we settle the hyperelliptic case: let $A \rightarrow \mathcal{M}_{0,2 g+2}$ be the jacobian of the universal hyperelliptic curve of genus $g>0$. By Torelli's theorem, one has $\operatorname{dim} \mu_{A}(S)=2 g-1$.

2.3.3. Theorem. Let $S$ be a finite cover of $\mathcal{M}_{0,2 g+2}$ and $\xi: S \rightarrow A$ be any non-torsion section. Then $\mathrm{rk} \beta \geq 2 g$.

While this may be considered as a consequence of the previous theorem by [27] or [1], we offer an elementary proof based on Corollary 2.2.4 combined with an explicit computation of the Kodaira-Spencer map.

As mentioned above, a real version of the hyperelliptic case is of particular interest and is treated in section 9 (independently of the main body of the paper); this is relevant to an issue recently raised by Serre, who pointed out a missing justification in an old work of Robinson on confinement of conjugates of algebraic integers [32]. We realized that this issue, although concerning only real points, fits into the present context and can be treated considering Betti maps. For a point $\left.s=\left(s_{1}, \ldots, s_{2 g}\right) \in S=\mathbb{P}^{1} \backslash\{0,1, \infty\}\right)^{2 g}$ with pairwise distinct coordinates, consider the corresponding genus $g$ hyperelliptic curve

$$
y^{2}=x(x-1)\left(x-s_{1}\right) \cdots\left(x-s_{2 g}\right) .
$$

It has two points at infinity, denoted as $\infty^{+}, \infty^{-}$. Denoting again by $A \rightarrow S$ the jacobian scheme of the curve defined by the above equation, we consider the section $\xi$ associating to each $s \in S$ the class of the divisor $\infty^{+}-\infty^{-}$. The abelian scheme and the section are

\footnotetext{
${ }^{7}$ In a previous version, an explicit link of these issues with the "Ax-Schanuel conjecture" was pointed out. Very recently this conjecture has been proved, making thus possible to apply it to our problem, as done by Gao in the appendix.
} 
both defined over the reals, so that for each real point $s \in S(\mathbb{R})$ the value $\xi(s)$ is a real point of the abelian variety $A_{s}$. The tangent space LieA also inherits a real structure, and we can define the real version of the Betti map as a map $\beta_{\mathbb{R}}: \tilde{S}(\mathbb{R}) \rightarrow \mathbb{R}^{g}$. We prove that $\beta_{\mathbb{R}}$ is generically a surjection, thus filling the gaps related to Serre's question.

\section{THE BETTI MAP.}

3.1. Betti coordinates on an abelian variety. Let us first clarify the "constant case", i.e. when the base $S$ is a point. Given a complex abelian variety $A$, we identify its period lattice $\mathcal{L}:=H_{1}(A(\mathbb{C}), \mathbb{Z})$ with the kernel of the exponential map

$$
\exp _{A}: \text { Lie } A \rightarrow A \text {. }
$$

Given $\xi \in A(\mathbb{C})$, we denote by $\mathcal{L}_{\xi}$ the lattice in $\left(\mathcal{L} \otimes_{\mathbb{Z}} \mathbb{R}\right) \oplus \mathbb{R}$ consisting of pairs $(\ell \in$ Lie $A, m \in \mathbb{Z})$ such that $\exp _{A}(\ell)=m \xi$; it sits in an extension

$$
0 \rightarrow \mathcal{L} \rightarrow \mathcal{L}_{\xi} \stackrel{(\ell, m) \mapsto m \xi}{\rightarrow} \mathbb{Z} \xi \rightarrow 0 .
$$

We fix a splitting $\lambda: \mathbb{Z} \xi \rightarrow \mathcal{L}_{\xi}$ (i.e. a branch of the "abelian logarithm"). On the other hand, let us consider the first projection $\mathcal{L}_{\xi} \stackrel{(\ell, m) \mapsto \ell}{\rightarrow}$ Lie $A$ and its $\mathbb{R}$-linear extension $\mathcal{L}_{\xi} \otimes_{\mathbb{Z}} \mathbb{R} \rightarrow$ Lie A. Since the composed map

$$
\mathcal{L} \otimes_{\mathbb{Z}} \mathbb{R} \rightarrow \mathcal{L}_{\xi} \otimes_{\mathbb{Z}} \mathbb{R} \rightarrow \text { Lie } A
$$

is an isomorphism, we get a retraction $\mathcal{L}_{\xi} \otimes_{\mathbb{Z}} \mathbb{R} \rightarrow \mathcal{L} \otimes_{\mathbb{Z}} \mathbb{R}$, and the image of $\lambda(\xi) \otimes 1$ in $\mathcal{L} \otimes_{\mathbb{Z}} \mathbb{R}$ can be expressed, in terms of a basis $\gamma$ of $\mathcal{L}$, by $2 g$ real coordinates, the Betti coordinates of $\xi^{8}$.

In terms of abelian integrals, the Betti coordinates of $\xi$ are nothing but the $2 g$ real solutions $\beta_{i}$ of the system of $g$ inhomogeneous linear equations with complex coefficients

$$
\int_{0}^{\xi} \omega_{j}=\sum_{i=1}^{2 g} \beta_{i} \int_{\gamma_{i}} \omega_{j}, j=1, \ldots g .
$$

3.2. The Betti map attached to a section of an abelian scheme. As explained above, we are interested in the relative setting, that is, in the variation of the Betti coordinates in the context of a family of abelian varieties endowed with a section.

Let $S$ be a smooth connected complex algebraic variety, and let $A \stackrel{f}{\rightarrow} S$ be an abelian scheme of relative dimension $g$. Its Lie algebra Lie $A$ is a rank $g$ vector bundle on $S$.

Let $\xi: S \rightarrow A$ be a section of $f$. The above constructions extend as follows. Let $\tilde{S}$ be a universal covering of $S(\mathbb{C})$, with its canonical structure of complex analytic manifold. The kernel of $\exp _{A}$ is a locally constant sheaf on $S(\mathbb{C})$, which can be viewed as a constant lattice $\mathcal{L}$ on $\tilde{S}$. Similarly, one constructs an exact sequence of lattices on $\tilde{S}$

$$
0 \rightarrow \mathcal{L} \rightarrow \mathcal{L}_{\xi} \stackrel{(\ell, m) \mapsto m}{\rightarrow} \mathbb{Z} \xi \rightarrow 0
$$

and one fixes a splitting $\lambda: \mathbb{Z} \xi \rightarrow \mathcal{L}_{\xi}$.

Let $\tilde{S}^{\text {real }}$ be the real-analytic manifold underlying $\tilde{S}$, and let $\mathcal{O}_{\tilde{S}^{r e a l}}^{\mathbb{R}}\left(\right.$ resp. $\mathcal{O}_{\tilde{S}^{\text {real }}}$ ) be the sheaf of real-valued (resp. complex valued) real-analytic functions on $\tilde{S}$. The the composition $\mathcal{L} \otimes_{\mathbb{Z}} \mathcal{O}_{\tilde{S}^{\text {real }}}^{\mathbb{R}} \rightarrow \mathcal{L}_{\xi} \otimes_{\mathbb{Z}} \mathcal{O}_{\tilde{S}^{\text {real }}}^{\mathbb{R}} \rightarrow$ Lie $A \otimes_{O_{S}} \mathcal{O}_{\tilde{S}^{\text {real }}}$ is an isomorphism

\footnotetext{
${ }^{8}$ This terminology, due to D. Bertrand, refers to the fact that these are indeed real-analytic coordinates on any simply-connected domain in $A(\mathbb{C})$, and that $\mathcal{L}_{\xi}$ is nothing but the Betti realization of the 1-motive $[\mathbb{Z} \stackrel{1 \mapsto \xi}{\rightarrow} A]$ attached to $(A, \xi)$ in the sense of $[9, \mathrm{X}]$.
} 
(relative version of (3.3)), whence a canonical retraction $\mathcal{L}_{\xi} \otimes_{\mathbb{Z}} \mathcal{O}_{\tilde{S}_{\text {real }}}^{\mathbb{R}} \rightarrow \mathcal{L} \otimes_{\mathbb{Z}} \mathcal{O}_{\tilde{S} \text { real }}^{\mathbb{R}}$, and the image of $\lambda(\xi) \otimes 1$ as a global section of $\mathcal{L} \otimes_{\mathbb{Z}} \mathcal{O}_{\tilde{S}^{\text {real }}}^{\mathbb{R}}$ can be expressed, in terms of a basis $\underline{\gamma}$ of the lattice $\mathcal{L}$, by a real-analytic map

$$
\beta=\beta_{\lambda, \underline{\gamma}}: \quad \tilde{S}^{r e a l} \rightarrow \mathbb{R}^{2 g},
$$

which we call the Betti map associated to $(A, \xi)$, or to the smooth 1 -motive $\left[\mathbb{Z}^{1 \mapsto \xi} \rightarrow A\right]$. It is compatible with pull-back of $(A, \xi)$ by any morphism $S^{\prime} \rightarrow S$, with $S^{\prime}$ smooth connected.

Pondering the nature of the Betti map, it is clear that its very definition pertains to realanalytic geometry, but the main technical issues of this paper involve its subtle behaviour with respect to the complex-analytic and algebraic ambient features.

To begin with, the interpretation of $\beta$ in terms of abelian integrals being the same as in (3.4) ("real" and "complex" being understood as "real-valued" and "complex-valued" respectively), it shows that the fibers of $\beta$ are complex-analytic subvarieties of $\tilde{S}$ ( $c f .4 .2$ ). The non-empty pre-images of the Betti map are complex subvarieties of dimension $\geq$ $\operatorname{dim} S-\frac{1}{2} \mathrm{rk} \beta$ ( $c f$. [7, prop. 2.2]).

\section{From Betti to Kodaira-Spencer.}

In this section, we relate the derivative of the Betti map $\beta$ at an arbitrary point $\tilde{s} \in \tilde{S}$ to the corresponding value $\theta_{\xi}^{\varpi}(s)$ of the contracted Kodaira-Spencer map for some specific parameter $\varpi$ (depending on $\tilde{s}$ ). Although we work, as above, over a (smooth) complex algebraic variety $S$ for convenience, the metamorphoses of the derivative of the Betti map which we display do not actually require the algebraicity of $S$.

4.1. Setting and notation. Let $A \rightarrow S$ be an abelian scheme of relative dimension $g$ over a smooth connected algebraic $\mathbb{C}$-variety of dimension $d$. For any holomorphic function $g$ on the universal covering $\tilde{S}$ of $S$, we denote by $\bar{g}$ the conjugate antiholomorphic function.

The problems studied in this paper are Zariski-local on $S$, and insensitive to replacing $A$ by an isogenous abelian scheme. Therefore we may and shall assume henceforth that

i) $S$ is affine and admits a system of global (etale) coordinates $\left(z_{1}, \ldots, z_{d}\right)$; we take $\left(\partial_{1}=\frac{\partial}{\partial z_{1}}, \ldots, \partial_{d}=\frac{\partial}{\partial z_{d}}\right)$ as a basis of tangent vector fields to $S$, also viewed as a basis of holomorphic derivations of $\mathcal{O}(\tilde{S})$,

ii) the vector bundle Lie $A$ is trivial, i.e. $\Gamma$ Lie $A$ is a free $\Gamma \mathcal{O}_{S}$-module of rank $g$; its dual is canonically isomorphic to the coherent sheaf $\Omega_{A}$ on $S$ of invariant 1-forms (a.k.a. differentials of the first kind),

iii) the vector bundle $\mathcal{H}_{d R}^{1}(A / S)$ is trivial,

iv) $A$ is principally polarized.

We fix a basis $\left(\omega_{1}, \ldots, \omega_{g}\right)$ of global sections of $\Omega_{A} \cong$ Lie $A$, and complete it into a symplectic basis $\left(\omega_{1}, \ldots, \omega_{g}, \eta_{1}, \ldots, \eta_{g}\right)$ of $\mathcal{H}_{d R}^{1}(A / S)$ (which carries the symplectic form coming from the polarization).

We fix a symplectic basis $\left(\gamma_{1}, \ldots, \gamma_{2 g}\right)$ of $\mathcal{L}$.

We set $\Omega_{1}:=\left(\int_{\gamma_{i}} \omega_{j}\right)_{i, j=1, \ldots g}, \Omega_{2}:=\left(\int_{\gamma_{i+g}} \omega_{j}\right)_{i, j=1, \ldots g}, \Omega:=\left(\begin{array}{l}\Omega_{1} \\ \Omega_{2}\end{array}\right)$,

The entries of $\Omega$ are holomorphic on $\tilde{S}$, and the $g \times g$-matrices $\Omega_{1}, \Omega_{2}$ and the $2 g \times 2 g$ matrix $(\Omega, \bar{\Omega})$ are invertible at every point $\tilde{s} \in \tilde{S}$.

Finally, we fix a section $\xi$ of $A / S$ and a determination $\lambda$ of the abelian logarithm as in 3.1. We may and shall assume that

$v)$ the extension $\mathcal{H}_{d R}^{1}\left(\left[\mathbb{Z}^{1 \mapsto \xi} \rightarrow A\right] / S\right)$ of $\mathcal{H}_{d R}^{1}(A / S)$ by $\mathcal{O}_{S}$ is trivial. 
We set $\Lambda:=\left(\int_{0}^{\xi} \omega_{j}\right)_{j=1, \ldots g}$, a row of $g$ holomorphic functions on $\tilde{S}$.

4.2. From real-analytic to holomorphic maps. We write the Betti map $\beta=\beta_{\lambda, \underline{\gamma}}$ of the 1-motive $[\mathbb{Z} \stackrel{1 \mapsto \xi}{\rightarrow} A]$ (in the basis $\left(\lambda(\xi), \underline{\gamma}\right.$ ) of $\mathcal{L}_{\xi}$ ) as a row with $2 g$ entries: by definition, $\lambda(\xi) \equiv \sum_{1}^{2 g} \beta_{i} \gamma_{i}$ in $\mathcal{L}_{\mathbb{R}}$, so that

$$
\Lambda=\beta \Omega .
$$

In particular, the fibers $\beta^{-1}(b)$ of $\beta$ are the complex-analytic subvarieties of $\tilde{S}$ defined by $\Lambda(\tilde{s})=b \Omega(\tilde{s})$. From (4.1), one gets $(\Lambda, \bar{\Lambda})=\beta(\Omega, \bar{\Omega})$ since $\beta$ is real-valued, whence

$$
\beta=(\Lambda, \bar{\Lambda})(\Omega, \bar{\Omega})^{-1}
$$

In particular, the entries of $\operatorname{det}(\Omega, \bar{\Omega}) \cdot \beta$ are polynomials in holomorphic and antiholomorphic functions on $\tilde{S}$.

It will be convenient to build the Jacobian matrix $J=J_{\lambda, \gamma}$ of the Betti map using the system of derivations $\left(\partial_{1}, \ldots, \partial_{d}, \bar{\partial}_{1}=\frac{\partial}{\partial \bar{z}_{1}}, \ldots, \bar{\partial}_{d}=\frac{\partial}{\partial \bar{z}_{d}}\right)$ :

$$
J=\left(\begin{array}{ccc}
\partial_{1} \beta_{1} & \ldots & \partial_{1} \beta_{2 g} \\
\vdots & & \vdots \\
\bar{\partial}_{d} \beta_{1} & \ldots & \bar{\partial}_{d} \beta_{2 g}
\end{array}\right) .
$$

Differentiating (4.2), one gets

$$
\partial_{i} \beta(\Omega, \bar{\Omega})=\left(\partial_{i} \Lambda, 0\right)-\beta\left(\partial_{i} \Omega, 0\right), \quad \bar{\partial}_{i} \beta(\Omega, \bar{\Omega})=\left(0, \bar{\partial}_{i} \bar{\Lambda}\right)-\beta\left(0, \bar{\partial}_{i} \bar{\Omega}\right) .
$$

For any $\nu \in \mathbb{C}^{2 g}$, let $I_{\nu}$ be the $d \times g$-matrix with holomorphic entries

$$
\left(I_{\nu}\right)_{i j}:=\partial_{i} \Lambda_{j}+\sum_{k} \nu_{k} \partial_{i} \Omega_{k j}
$$

Combining equations (4.4) for $i=1, \ldots, d$, one gets

$$
J(\tilde{s}) \cdot(\Omega, \bar{\Omega})(\tilde{s})=\left(\begin{array}{cc}
I_{-\beta(\tilde{s})} & 0 \\
0 & \bar{I}_{-\beta(\tilde{s})}
\end{array}\right)(\tilde{s}),
$$

so that for every $s \in \tilde{S}$,

$$
\operatorname{rk} J(\tilde{s})=\operatorname{rk} J(\tilde{s})(\Omega, \bar{\Omega})(\tilde{s})=2 \operatorname{rk} I_{-\beta(\tilde{s})}(\tilde{s}) .
$$

In particular, rk $J(\tilde{s})$ is even.

4.2.1. Remarks. (1) Just like $\operatorname{det}(\Omega, \bar{\Omega}) \cdot \beta$, the entries of $\operatorname{det}(\Omega, \bar{\Omega}) I_{-\beta}$ are polynomials in holomorphic and antiholomorphic functions on $\tilde{S}$.

2) On the other hand, the entries of $I_{-\beta}$ are monodromy-invariant (hence real-analytic functions on $S$ ). Indeed, there is a natural action of the deck transformation group $\Delta:=$ $\operatorname{Aut}(\tilde{S} / S)$ on $\mathcal{L}_{\xi}$ which preserves $\mathcal{L}$, and for any $\delta \in \Delta$, one can write $\delta \underline{\gamma}=M_{\delta} \underline{\gamma}$ and $\delta \lambda(\xi)=\lambda(\xi)+\sum_{k} \nu_{\delta, k} \gamma_{k}$ (where the entries of $M_{\delta}$ and $\nu_{\delta}$ are integers), whence (by integration of holomorphic differentials along these cycles)

$$
\Omega(\delta \tilde{s})=M_{\delta} \Omega(\tilde{s}), \Lambda(\delta \tilde{s})=\Lambda(\tilde{s})+\nu_{\delta} \Omega(\tilde{s}) .
$$

Using (4.2), one gets $\beta_{\lambda, \underline{\gamma}}(\tilde{s})+\nu=\left(\beta_{\delta \lambda, \delta \underline{\gamma}}(\delta \tilde{s})\right) M_{\delta}$, whence

$$
J_{\lambda, \underline{\gamma}}(\tilde{s})=J_{\delta \lambda, \delta \underline{\gamma}}(\delta \tilde{s}) M_{\delta}, \quad I_{-\beta(\tilde{s})}(\delta \tilde{s})=I_{-\beta(\tilde{s})}(\tilde{s}) .
$$


This shows that $r k \beta(\tilde{s})$ depends only on the point $s \in S$ under $\tilde{s}$. In fact the same calculation shows that it does not even depend on the auxiliary choice of $(\lambda, \underline{\gamma})$.

4.3. Going to the Siegel space $\mathfrak{H}_{g}$. We set $Z:=\Omega_{2} \cdot \Omega_{1}^{-1}, \quad L:=\Lambda \cdot \Omega_{1}^{-1}$ and note that $Z$ takes values in $\mathfrak{H}_{g}$, i.e. $Z$ is symmetric and $\Im Z>0$ (where $\Im$ stands for the imaginary part). From $\Lambda=\beta \Omega$, one gets

$$
\beta=(L, \bar{L})\left(\begin{array}{ll}
I & I \\
Z & \bar{Z}
\end{array}\right)^{-1}
$$

(where $I$ stands for the $g \times g$ identity matrix), whence $\beta=\left(\beta_{1}, \beta_{2}\right)$ with

$$
\begin{gathered}
\beta_{1}+\beta_{2} Z=L, \\
\beta_{2}=\Im L(\Im Z)^{-1}, \beta_{2}=\frac{1}{2}\left(-L(\Im Z)^{-1} \bar{Z}+\bar{L}(\Im Z)^{-1} Z\right) .
\end{gathered}
$$

For any $\mu \in \mathbb{C}^{g}$, let $H_{\mu}$ be the $d \times g$-matrix with holomorphic entries

$$
\left(H_{\mu}\right)_{i j}:=\partial_{i} L_{j}+\sum_{k} \mu_{k} \partial_{i} Z_{k j}
$$

On denoting by $\beta(\tilde{s})_{1}$ the first half of the row $\beta(\tilde{s})$, one draws as above (cf. (4.3) (4.6)):

$$
J(\tilde{s}) \cdot\left(\begin{array}{ll}
I & I \\
Z & \bar{Z}
\end{array}\right)(\tilde{s})=\left(\begin{array}{cc}
H_{-\beta(\tilde{s})_{1}} & 0 \\
0 & \bar{H}_{-\beta(\tilde{s})_{1}}
\end{array}\right)(\tilde{s}) .
$$

A straightforward calculation shows that

$$
\partial \Lambda+\nu \partial \Omega=\left(\partial L+\nu_{2} \partial Z\right) \Omega_{1}+\left(L+\nu_{1}+\nu_{2} Z\right) \partial \Omega_{1},
$$

so that

$$
H_{\nu_{2}}(\tilde{s}) \cdot \Omega_{1}(\tilde{s})=I_{\nu}(\tilde{s})
$$

if $\nu=\left(\nu_{1}=-L(\tilde{s})-\nu_{2} Z(\tilde{s}), \nu_{2}\right)$, i.e. if $\nu_{1} \Omega_{1}+\nu_{2} \Omega_{2}=-\Lambda$. For $\nu=-\beta(\tilde{s})$, one recovers (4.14) from (4.6) using (4.11); more generally,

$$
\max _{\nu \in \mathbb{C}^{2 g}} \operatorname{rk} I_{\nu}(\tilde{s}) \geq \max _{\mu \in \mathbb{C}^{g}} \operatorname{rk} H_{\mu}(\tilde{s})
$$

4.4. From holomorphic to rational functions. We set $M:=\left(\int_{0}^{\xi} \eta_{j}\right)_{j=1, \ldots g}$.

The $(1+2 g) \times(1+2 g)$-matrix $Y_{\xi}:=\left(\begin{array}{ccc}1 & \Lambda & M \\ 0 & \Omega_{1} & N_{1} \\ 0 & \Omega_{2} & N_{2}\end{array}\right)$ is a full solution of the GaussManin connection attached to the family of 1 -motives $\left[\mathbb{Z}^{1 \mapsto \xi} \rightarrow\right.$ $A$, which has the form ${ }^{9}$

$$
\partial Y_{\xi}=Y_{\xi}\left(\begin{array}{ccc}
0 & \ell_{\partial} & m_{\partial} \\
0 & R_{\partial} & S_{\partial} \\
0 & T_{\partial} & U_{\partial}
\end{array}\right)
$$

where the entries of the last displayed matrix lie in $\mathcal{O}(S)$, and depend linearly on the tangent vector field $\partial \in \Gamma T_{S}$. The advantage of going to the Siegel space, i.e. of considering $H_{\mu}$ rather than $I_{\nu}$, appears in the following calculations based on (4.18):

$$
(\partial L) \cdot \Omega_{1}=\ell_{\partial}+(M-\Lambda N) T_{\partial}
$$

\footnotetext{
9 as in [4], we write this differential system in a slightly non-standard way, with the matrix of the connection on the right so that the monodromy acts on the left. $\ell_{\partial}$ is essentially the row of rational functions which occur as second member of the inhomogeneous scalar differential operator in Manin's kernel theorem.
} 
while

$$
\begin{aligned}
& (\partial Z) \cdot \Omega_{1}=\partial \Omega_{2}-\Omega_{2} \Omega_{1}^{-1} \partial \Omega_{1}=\left(\Omega_{2} R_{\partial}+N_{2} T_{\partial}\right)-\Omega_{2} \Omega_{1}^{-1}\left(\Omega_{1} R_{\partial}+N_{1} T_{\partial}\right) \\
& \quad=\left(N_{2}-\Omega_{1} \Omega_{1}^{-1} N_{1}\right) T_{\partial}={ }^{t} \Omega_{1}^{-1}\left({ }^{t} \Omega_{1} N_{2}-{ }^{t} \Omega_{2} N_{1}\right) T_{\partial}=2 \pi i^{t} \Omega_{1}^{-1} T_{\partial}
\end{aligned}
$$

(using the symmetry of $Z$ and, at the end, the analogue of the Legendre relation for abelian varieties).

Let $s$ be the image of $\tilde{s}$ in $S$. For any $\mu \in \mathbb{C}^{g}$, let $G_{\mu}$ be the $d \times g$-matrix with rational entries

$$
\left(G_{\mu}\right)_{i j}:=\left(\ell_{\partial_{i}}\right)_{j}+\sum_{k} \mu_{k}\left(T_{\partial_{i}}\right)_{k j} \in \mathcal{O}(S) .
$$

It follows from (4.19) and (4.20) that

$$
H_{\mu} \Omega_{2}=G_{M-\Lambda N+2 \pi i \mu}{ }^{t} \Omega_{1}^{-1}
$$

and since for fixed $\tilde{s}, \mu \mapsto M(\tilde{s})-\Lambda(\tilde{s}) N(\tilde{s})+2 \pi i \mu^{t} \Omega_{1}^{-1}(\tilde{s})$ is an affine automorphism of $\mathbb{C}^{g}$, one concludes that

$$
\max _{\mu \in \mathbb{C}^{g}} \operatorname{rk} H_{\mu}(\tilde{s})=\max _{\mu \in \mathbb{C}^{g}} \operatorname{rk} G_{\mu}(s)
$$

4.5. $G_{\mu}$ and the Kodaira-Spencer map. Recall that for any variation $\underline{\mathcal{V}}=\left(\mathcal{V}, \mathcal{F}^{i}, \nabla\right)$ of (mixed) Hodge structures on $S$, the Griffiths transversality condition $\nabla\left(\mathcal{F}^{i}\right) \subset \mathcal{F}^{i-1}$ implies that the (Gauss-Manin) connection $\nabla$ induces $\mathcal{O}_{S}$-linear maps (the Kodaira-Spencer maps) $\theta^{i}: T_{S} \otimes g r^{i} \rightarrow g r^{i-1}$, where $g r^{i}=\mathcal{F}^{i} / \mathcal{F}^{i+1}$ are the graded pieces of the Hodge filtration $(c f .[18,1.3])$. In case $\underline{\mathcal{V}}$ comes from an abelian scheme or a 1 -motive over $S$, the Hodge filtration has only two steps: $g r^{1}$ and $g r^{0}$, so that there is only one Kodaira-Spencer $\operatorname{map} \theta: T_{S} \otimes g r^{1} \rightarrow g r^{0}$ (cf. also [4, 1.4]).

Let us consider the family of 1 -motives $\left[\mathbb{Z}^{1 \mapsto \xi} \rightarrow A\right]$ defined by $(A, \xi)[9, \mathrm{X}]$ : in this case, $\mathcal{V}=\mathcal{H}_{d R}^{1}\left(\left[\mathbb{Z}^{1 \mapsto \xi} \rightarrow\right] / S\right)$ is a vector bundle of rank $2 g+1$, and $g r^{1}=\Omega_{A}$. The associated Kodaira-Spencer map is a morphism of vector bundles

$$
\theta_{\xi}: T_{S} \otimes \Omega_{A} \rightarrow g r^{0}
$$

whose composition with the canonical projection $g r^{0} \rightarrow$ Lie $A$ is the usual KodairaSpencer map of $A$ :

$$
\theta_{A}: T_{S} \otimes \Omega_{A} \rightarrow \Omega_{A}^{\vee}=\text { Lie A. }
$$

Conditions $i i i), i v), v$ ) above imply that the Hodge-theoretic graded piece $g r^{0}:=$ $g r^{0} \mathcal{H}_{d R}^{1}\left(\left[\mathbb{Z}^{\stackrel{1 \mapsto \xi}{\rightarrow}} A\right] / S\right)$ is a trivial extension of Lie $A$ by $\mathcal{O}_{S}$. We denote by $\omega_{0}$ a lifting of 1 in $\left(g r^{0}\right)^{\vee}$, so that $\left(\omega_{0}, \omega_{1}, \ldots, \omega_{g}\right)$ form a basis of sections of $\left(g r^{0}\right)^{\vee}$.

Using the basis $\left(\omega_{1}, \ldots, \omega_{g}\right)$ and the dual basis ( $c f$. Notation (4.18)), it turns out that the matrix of the contracted map $\theta_{A, \partial}: \Omega_{A} \rightarrow \Omega_{A}^{\vee}$ is nothing but the symmetric matrix $T_{\partial}, c f .[4,1.4]$. Similarly, the matrix of the contracted map $\theta_{\xi, \partial}: \Omega_{A} \rightarrow g r^{0}$ is $\left(\begin{array}{l}\ell_{\partial} \\ T_{\partial}\end{array}\right)$. It follows that $G_{\mu}$ is the matrix of the contracted map

$$
\theta_{\xi}^{\varpi}: T_{S} \rightarrow \Omega_{A}^{\vee}=\text { Lie } A, \quad \varpi=\sum_{k=0}^{g} \mu_{k} \omega_{k} \in\left(g r^{0}\right)^{\vee}, \mu_{0}=1 .
$$


Combining (4.6), (4.16) and (4.22), one finally gets

$$
J(\tilde{s}) \cdot(\Omega, \bar{\Omega})(\tilde{s})=\left(\begin{array}{cc}
\theta_{\xi}^{\varpi(\tilde{s})}(s) & 0 \\
0 & \bar{\theta}_{\xi}^{\varpi(\tilde{s})}(s)
\end{array}\right)
$$

with $\varpi(\tilde{s})=\omega_{0}+\sum_{k=1}^{g}\left(M-\Lambda N+2 \pi i \beta_{2}{ }^{t} \Omega_{1}^{-1}\right)_{k}(\tilde{s}) \cdot \omega_{k}$, and $\beta_{2}$ given by (4.12); whence

$$
\operatorname{rk} J(\tilde{s}) \leq 2 \max _{\varpi} \operatorname{rk} \theta_{\xi}^{\varpi}(s) .
$$

Combining (4.7), (4.17) and (4.23), one also gets

$$
\max _{\varpi} \operatorname{rk} \theta_{\xi}^{\varpi}(s) \leq \max _{\nu \in \mathbb{C}^{2 g}} \operatorname{rk} I_{\nu}(\tilde{s}) .
$$

\section{GENERIC RANK OF THE BETTI MAP.}

In this section, we show that the generic rank of the (derivative of the) Betti map is twice the generic rank of the contracted Kodaira-Spencer map $\theta_{\xi}^{\varpi}$ for a generic value of the parameter $\varpi$. Here, the algebraicity of $S$ is essential, as well as the assumptions of Th. 2.2.1:

(*) $\mathbb{Z} \xi$ is Zariski-dense in $A$, and $A$ has no fixed part.

\subsection{A strengthened form of Theorem 2.2.3.}

5.1.1. Theorem. Under $(*) \operatorname{rk} J=2 \cdot \max _{\nu \in \mathbb{C}^{2 g}} \operatorname{rk} I_{\nu}$.

Here, the ranks are the generic ranks. This implies the formula $\mathrm{rk} J=2 \max _{\varpi} \operatorname{rk} \theta_{\xi}^{\varpi}$ of Theorem 2.2.3, due to (4.28) and (4.29). This also implies Theorem 2.2.1, thanks to (4.14) (or (4.17)).

Inequality $\leq$ in Theorem 5.1 .1 follows from (4.7). If $\mathrm{rk} \beta$ takes its maximal possible value $2 \min (d, g)$, we have equality since $\operatorname{rk} I_{\nu} \leq \min (d, g)$. Hence we may assume that

(**) $\quad r:=\operatorname{rk} J<2 \min (d, g)$.

Equivalently (by (4.7)): every minor $\mathcal{M}_{-\beta}$ of order $r+1$ in $I_{-\beta}$ vanishes identically on $\tilde{S}$. We have to show that every minor $\mathcal{M}_{\nu}$ of order $r+1$ in $I_{\nu}$ vanishes identically on $\tilde{S}$ for every $\nu \in \mathbb{C}^{2 g}$.

5.2. Separation of the holomorphic and antiholomorphic variables. By Remark 4.2.1 $(1), \operatorname{det}(\Omega, \bar{\Omega})^{r} \mathcal{M}_{-\beta}$ is a polynomial in holomorphic functions and antiholomorphic functions on $\tilde{S}$, i.e. lies in the image of the "diagonal" ring homomorphism

$$
\iota: \mathcal{O}(\tilde{S}) \otimes \overline{\mathcal{O}}(\tilde{S}) \rightarrow \mathcal{O}\left(\tilde{S}^{\text {real }}\right), \quad f(z) \bar{g}(w) \mapsto f(z) \bar{g}(z) .
$$

5.2.1. Lemma. $\iota$ is injective.

Proof. If $\sum f_{i}(z) \bar{g}_{i}(z)=0$, then $\sum f_{i}(z) g_{i}(w) \in \mathcal{O}(\tilde{S} \times \tilde{S})$ vanishes on the real-analytic subvariety of $\tilde{S} \times \tilde{S}$ given by the equation $z=\bar{w}$, hence on the smallest complex-analytic subvariety which contains it, which is $\tilde{S} \times \tilde{S}$ (the computation being local, one may replace $\tilde{S}$ by a open subset of $\mathbb{C}^{d}$; looking at tangent spaces, the result follows by induction on $d$ ).

In fact, $\operatorname{det}(\Omega, \bar{\Omega})^{r} . \mathcal{M}$ can be written as a polynomial $P$ in the components of $\Lambda, \partial \Lambda, \Omega, \partial \Omega$ and their complex-conjugates. By the previous lemma, one has

$$
P\left(\Lambda(\tilde{s}), \partial \Lambda(\tilde{s}), \Omega(\tilde{s}), \partial \Omega(\tilde{s}), \bar{\Lambda}\left(\tilde{s}^{\prime}\right), \overline{\partial \Lambda}\left(\tilde{s}^{\prime}\right), \bar{\Omega}\left(\tilde{s}^{\prime}\right), \overline{\partial \bar{\Omega}}\left(\tilde{s}^{\prime}\right)\right)=0
$$

for any pair $\left(\tilde{s}, \tilde{s}^{\prime}\right) \in \tilde{S}^{2}$. 
5.3. Taking advantage of the monodromy: from a single equation to a parametrized system of equations. If we temporarily fix $\tilde{s}^{\prime}$, (5.2) becomes an identity in $\mathbb{C}[\Lambda, \partial \Lambda, \Omega, \partial \Omega]$. In particular, it is invariant under the monodromy group, which acts on $\Lambda$ and $\Omega$ by the formulas (4.8) (which are independent of $\tilde{s}^{\prime}$ ). It is also invariant under its Zariski closure, which coincides with the differential Galois group of (4.18).

According to [2, Th. 3], under assumption $(*)$, the kernel of the map of algebraic monodromy groups $\left\{\left(\begin{array}{cc}1 & \nu_{\delta} \\ 0 & M_{\delta}\end{array}\right)\right\}_{\delta \in \Delta}^{Z a r} \rightarrow\left\{M_{\delta}\right\}_{\delta \in \Delta}^{Z a r}$ is Zariski-dense in $\mathbb{C}^{2 g}$ (this result can be interpreted as a theorem of linear independence of abelian logarithms with respect to periods, $c f$. also $[3, \S 1]$ for a more concise and transparent proof Therefore, if one replaces $(\Lambda, \partial \Lambda)$ by $(\Lambda+\nu \Omega, \partial \Lambda+\nu \partial \Omega)$ for any $\nu \in \mathbb{C}^{2 g},(5.2)$ still holds.

5.3.1. Remarks. (1) [2, Th. 3] relies on the "theorem of the fixed part" for the variation of mixed Hodge structures attached to the 1 -motive $[\mathbb{Z} \stackrel{\xi}{\rightarrow} A]$ over $S$. In [2], this property is checked by proving that the variation is "good" (which is established in lemma 5 of loc. cit. when $S$ is a curve, and one reduces to this case restricting to a sufficiently general curve on $S$, or alternatively using the fact that "goodness" may be checked in dimension 1 , cf. also [37, Th. 2.2]), and then applying the theorems of Steenbrink-Zucker-Schmid about good variations of mixed Hodge structures.

Here is a sketch of a much simpler proof of the "theorem of the fixed part" in this case: passing to the Cartier dual of $[\mathbb{Z} \stackrel{\xi}{\rightarrow} A]$, one deals instead with a semi-abelian scheme, which allows one to conclude by the standard Deligne-Katz argument, using a suitable relative compactification [18, 4.3.4].

(2) [2, Th. 3] deals with relative algebraic monodromy. It is not clear whether the same maximality property holds for relative monodromy before taking Zariski closures ${ }^{10}$ (this is an interesting open problem, but unrelated to our problem).

(3) Equation (5.2) expresses the annulation of an (arbitrary) minor of $\iota^{-1}\left(I_{-\beta} \cdot \operatorname{det}(\Omega, \bar{\Omega})\right)$ ). If one replaces $(\Lambda, \partial \Lambda)$ by $(\Lambda+\nu \Omega, \partial \Lambda+\nu \partial \Omega)$, then (by (4.2)) $\iota^{-1}(\beta)$ becomes $\iota^{-1}(\beta)+$ $\nu \iota^{-1}\left((\Omega, \bar{\Lambda})(\Omega, \bar{\Omega})^{-1}\right)$, and $\left.\iota^{-1}\left(I_{-\beta} \cdot \operatorname{det}(\Omega, \bar{\Omega})\right)\right)$ becomes $\left.\iota^{-1}\left(I_{\breve{\nu}} \cdot \operatorname{det}(\Omega, \bar{\Omega})\right)\right)$, with $\breve{\nu}=\left(0, \nu \bar{\Omega}\left(\tilde{s}^{\prime}\right)\right)\left(\Omega(\tilde{s}), \bar{\Omega}\left(\tilde{s}^{\prime}\right)\right)^{-1}$. Since $\breve{\nu}$ depends only on $g$ parameters (instead of $2 g$ ), this is not enough to conclude. This is why we have to "double" the monodromy action, using Lemma 5.1.

Instead of keeping $\tilde{s}^{\prime}$ fixed, we will use monodromy of the second factor as well (and simultaneously), i.e. the action of $\pi_{1}\left(S \times S,\left(s, s^{\prime}\right)\right) \cong \pi_{1}(S, s) \times \pi_{1}\left(S^{\prime}, s^{\prime}\right)$ (up to complex-conjugation on the second factor, this amounts to applying [2, Th. 3] in the product situation $\left.\left[\mathbb{Z}^{2} \rightarrow A^{2}\right] / S^{2}\right)$. On the second factor, we have to replace $\left(\bar{\Lambda}, \overline{\partial \Lambda^{\prime}}\right)$ by $\left(\bar{\Lambda}+\nu^{\prime} \bar{\Omega}, \overline{\partial \bar{\Lambda}}+\nu^{\prime} \bar{\partial}\right)$ for any $\nu^{\prime} \in \mathbb{C}^{2 g}$. Then $\iota^{-1}\left(I_{-\beta} \cdot \operatorname{det}(\Omega, \bar{\Omega})\right)$ becomes $\iota^{-1}\left(I_{\breve{\nu}} \cdot \operatorname{det}(\Omega, \bar{\Omega})\right)$, with

$$
\breve{\nu}=\left(\nu^{\prime} \Omega(\tilde{s}), \nu \bar{\Omega}\left(\tilde{s}^{\prime}\right)\right)\left(\Omega(\tilde{s}), \bar{\Omega}\left(\tilde{s}^{\prime}\right)\right)^{-1} .
$$

For fixed $\left(\tilde{s}, \tilde{s}^{\prime}\right)$, it is then clear that $\breve{\nu}$ can take arbitrary values in $\mathbb{C}^{2 g}$ when $\left(\nu, \nu^{\prime}\right)$ varies in $\mathbb{C}^{4 g}$. One concludes that every minor $\mathcal{M}_{\breve{\nu}}$ of order $r+1$ in $I_{\breve{\nu}}$ vanishes identically on $\tilde{S}$ for every $\breve{\nu} \in \mathbb{C}^{2 g}$ as wanted. This finishes the proof of Theorems 5.1.1 and 2.2.3.

Proof of Cor. 2.2.2. In this situation $d \geq g$. As we have seen, by (4.14), Theorem 5.1.1 implies that $\mathrm{rk} J=2 \cdot \max _{\mu \in \mathbb{C}^{g}} \operatorname{rk} H_{\mu}$. If $J$ is nowhere submersive, we have rk $J<2 g$, hence

\footnotetext{
10 in this respect, the presentation of $[2$, Th. 3] in $[36, \S 3]$ is an overinterpretation.
} 
for any $\mu, \operatorname{rk} H_{\mu}<g$. This amounts to saying that for any $\left(\mu_{0}, \mu_{1}, \ldots, \mu_{g}\right) \in \mathbb{C}^{g+1}$, all $g$-g-minors of the matrix with entries $\mu_{0} \partial_{i} L_{j}+\sum_{k} \mu_{k} \partial_{i} Z_{k j}$ vanish. For $\mu_{0}=0$, consider the anaytic map sending $\tilde{s} \in \tilde{S}$ to $Z(\tilde{s}) . \mu \in \mathbb{C}^{g}$. Its (complex-analytic) rank is $<g$, hence its non-empty fibers are complex analytic subvarieties of dimension $\geq \operatorname{dim} S+1-g$.

5.3.2. Remarks. (1) Assumption $(*)$ is essential for 5.1.1: for instance, it is known that $\theta_{A}=0$ if and only if $A$ becomes constant on a finite etale covering (cf. [4, 1.4 .2 ii]), and by the same argument, $\theta_{\xi}=0$ (equivalently: $\theta_{\xi}^{\varpi}=0$ for all $\varpi$ ) if and only if $(A, \xi)$ becomes constant on a finite etale covering; on the other hand, $J=0$ whenever $\xi$ is a torsion section, even if $A$ is non isotrivial.

(2) When $d=g$, Theorem 2.2.1 expresses the condition " $\beta$ is nowhere submersive" in the form: for any $\tilde{s},\left(\partial_{i} L_{j}(\tilde{s})\right)$ and $\left(\partial_{i} Z_{k j}(\tilde{s})\right), k=1, \ldots, g$, span a vector space of singular matrices. One recognizes a special case of the notoriously difficult Dieudonné problem: describe vector spaces of singular (symmetric) matrices, $c f .[22]^{11}$.

(3) Given an abelian scheme $A / S$, the vector bundle $\Omega_{A}$ on $S$ is endowed with a $\mathcal{D}_{S^{-}}$ module structure by the Gauss-Manin connection. The Kodaira-Spencer map $\theta_{A}$ is the $\mathcal{O}_{S}$-linear map $T_{S} \otimes \Omega_{A} \rightarrow \mathcal{H}_{\mathrm{dR}}^{1}(A / S) / \Omega_{A}$ induced by the Gauss-Manin connection. It is then natural to introduce and compare the following 'ranks', as done in [4]:

$$
\begin{aligned}
& r=r(A / S)=\operatorname{rk} \mathcal{D}_{S} \Omega_{A} / \Omega_{A}, \\
& r^{\prime}=r^{\prime}(A / S)=\operatorname{rk} \theta=\operatorname{rk} \mathcal{D}_{S}^{\leq 1} \Omega_{A} / \Omega_{A}, \\
& r^{\prime \prime}=r^{\prime \prime}(A / S)=\max _{\partial} \operatorname{rk} \theta_{\partial} .
\end{aligned}
$$

One always has $r^{\prime \prime} \leq r^{\prime} \leq r \leq g$. Usually, by rank of the Kodaira-Spencer map one means the integer $r^{\prime}$, and one says that the Kodaira-Spencer map is non-degenerate if $r^{\prime}=g$, which amounts to the condition [4, Lemma 1.4.5]

$$
\forall \omega \neq 0, \exists \partial, \quad \theta_{A, \partial} \omega \neq 0 .
$$

On the other hand, for $d=g$, the hypothesis of our Cor. 2.2.4 reads:

$$
\exists \omega, \forall \partial \neq 0, \quad \theta_{A, \partial} \omega \neq 0 .
$$

There is no implication between (5.4) and (5.5) in either direction in general (but we shall see that the conditions are related if $g \leq 3$ ).

\section{Abelian schemes With $E n d_{S} A=\mathbb{Z}$ AND $d_{\mu_{A}} \geq g$. MAin TheOrem.}

6.1. In this section, we prepare the proof of Theorem 2.3.1 by reducing to the case of maximal monodromy, the proof of which is treated in detail in App. 2.

6.1.1. Theorem. Let $A \rightarrow S$ be a principally polarized abelian scheme of relative dimension $g$, such that

(1) A has no non-trivial endomorphism over any finite covering of $S$,

(2) $d_{\mu_{A}}:=\operatorname{dim} \operatorname{Im}\left(\mu_{A}: S \rightarrow \mathcal{A}_{g}\right)$ is at least $g$.

Then the monodromy of $A \rightarrow S$ is Zariski-dense in $S p_{2 g}$.

Proof. One uses the representation-theoretic classification of abelian varieties $B$ with End $B=\mathbb{Z}$ given by Borovoi [6] (which one applies to a general fiber $B$ of $A \rightarrow S$ ). He proves that the special Mumford-Tate group is $\mathbb{Q}$-simple, and more precisely has the form $G=\operatorname{Res}_{F / \mathbb{Q}} H$ where $F$ is a totally real number field and $H$ is an absolutely simple

\footnotetext{
${ }^{11}$ we are grateful to M. Brion for this reference.
} 
$F$-group. Thus $G_{\mathbb{R}}$ decomposes as a product $\Pi_{1}^{m} H_{i}$ of absolutely simple real groups (obtained from $H$ at the various real places of $F$ ), and it follows from Satake's classification that only $H_{1}$ is non-compact (up to permutation). Accordingly, $H^{1}(B, \mathbb{C})$ decomposes as a tensor product $\otimes V_{i}$ where each $V_{i}$ is an irreducible symplectic $\mathbb{C}$-representation of $H_{i \mathbb{C}}$. They are conjugate under the Galois group of $F^{g a l} / \mathbb{Q}$, and in particular have the same dimension $2 d$, so that

$$
(2 d)^{m}=2 g,
$$

and $m$ is odd (again by the symplectic condition).

In order to prove the theorem, one may assume that that $A[n]$ is constant for some $n$, and relplace $S$ by the smallest special subvariety of $\mathcal{A}_{g, n}$ containing $S$, and $A$ by the universal abelian scheme over it (cf. e.g. App. 2. Lemma 2.6). The conclusion of the theorem amounts to saying that generic Mumford-Tate group is $G S p_{2 g}$, or equivalently that the special Mumford-Tate group $G$ of a general fiber $B$ is $S p_{2 g}$.

It is known that, taking the above notation, $H_{1}$ is actually a classical absolutely simple $\mathbb{R}$-group: in particular, $H_{1 \mathbb{C}}$ belongs to one of the series $A_{\ell}, B_{\ell}, C_{\ell}, D_{\ell}$ (with $\ell \geq 2,3,1,4$ respectively ${ }^{12}$ ), and accordingly the hermitian symmetric domain $X_{1}=\tilde{S}$ belongs to one of the series $X_{A_{\ell}}=S U(r, \ell+1-r) / S(U(r) \times U(\ell+1-r)), r \in\{1, \ldots, \ell\}$,

$$
\begin{gathered}
X_{B_{\ell}}=S O(2,2 \ell-1) / S O(2) \times S O(2 \ell-1), \\
X_{C_{\ell}}=\mathfrak{H}_{\ell}, \text { the Siegel space, } \\
X_{D_{\ell}^{\mathbb{R}}}=S O(2,2 \ell-2) / S O(2) \times S O(2 \ell-2),
\end{gathered}
$$

"quaternionic version" of the latter, $X_{D_{\ell}^{\mathbb{H}}}=S O(2 \ell)^{\mathbb{H}} / U(\ell)$.

Their real dimensions are respectively

$$
2 r(\ell+1-r), 2(2 \ell-1), \ell(\ell+1), 4(\ell-1), \ell(\ell-1) .
$$

For such $A \rightarrow S$, condition (2) in the theorem means that $\operatorname{dim}_{\mathbb{R}} X_{1} \geq 2 g$, which becomes in each case:

$$
\begin{gathered}
2 r(\ell+1-r) \geq(2 d)^{m}, 2(2 \ell-1) \geq(2 d)^{m}, \ell(\ell+1) \geq(2 d)^{m}, \\
4(\ell-1) \geq(2 d)^{m}, \ell(\ell-1) \geq(2 d)^{m} .
\end{gathered}
$$

To relate $\ell$ and $d$, one needs one more piece of information about the representation $V_{1 \mathbb{C}}$ : according to Deligne, it is minuscule [10]. The classification of minuscule representations shows that in case $A_{\ell}, V_{1 \mathbb{C}}$ is a wedge power $\wedge^{i} V_{s t}$ of the standard representation, in case $B_{\ell}$ the spin representation of dimension $2 d=2^{\ell}$, in case $C_{\ell}$ the standard representation $V_{s t}$ of dimension $2 d=2 \ell$, in case $D_{\ell}$ either the standard representation or the half-spin representations of dimension $2 d=2^{\ell-1}$.

The only symplectic cases among them are: in case $A_{\ell}, \wedge^{(\ell+1) / 2} V_{s t}$ if $\ell \equiv 1(4)$, in case $B_{\ell}$ the spin representation if $\ell \equiv 1,2,5,6(8)$, in case $C_{\ell}$ the standard representation $V_{s t}$, and in case $D_{\ell}$ the half-spin representations if $\ell \equiv 2(4)$ (cf. also Mustafin's table [28]). These conguences imply $\ell \geq 5$ in the $A_{\ell}$ and $B_{\ell}$ cases, and $\ell \geq 6$ in the $D_{\ell}$ case.

The above inegalities become

$$
\begin{gathered}
2 r(\ell+1-r) \geq\left(\begin{array}{c}
\ell+1 \\
(\ell+1) / 2
\end{array}\right)^{m}, 2(2 \ell-1) \geq 2^{\ell m}, \\
\ell(\ell+1) \geq(2 \ell)^{m}, 4(\ell-1) \geq 2^{(\ell-1) m}, \ell(\ell-1) \geq 2^{(\ell-1) m},
\end{gathered}
$$

with $m$ odd.

\footnotetext{
${ }^{12}$ more convenient here than the usual convention 1, 2, 3, 4: but $A_{1}=C_{1}$ and $B_{2}=C_{2}$.
} 
Let us first consider the third inequality ( $C_{\ell}$ case). Since $m$ is odd, it is fulfilled if and only if $m=1$; this corresponds to the usual universal family $\mathcal{X}_{g, n} \rightarrow \mathcal{A}_{g, n}$ with $g=\ell$ and $G=S p_{2 g}$. We claim that the other cases are impossible. For this, it suffices to consider $m=1$. A simple inspection shows that the first two inequalities are impossible for $\ell \geq 5$ (and any $r$ ), and the last two ones are impossible for $\ell \geq 6$.

6.2. Proof of Theorem 2.3.1. Theorem 6.1.1 reduces the proof of Theorem 2.3.1 to the special case where the Zariski-closure of the monodromy of the scheme $A \rightarrow S$ is the full symplectic group $S p_{2 g}$. In this situation, Gao's Theorem 10.1.1 shows that our Corollary 2.2.2 applies, and this ends the proof of Theorem 2.3.1.

6.3. An example. Beyond the case $E n d=\mathbb{Z}$, even for abelian scheme with simple geometric generic fiber, the condition in Cor. 2.2.4 may fail, in which case for any section $\xi$, our method fails to establish whether $\beta$ is generically a submersion.

Here is an example. Let $L^{+}$be a real quadratic field and $L$ a totally imaginary quadratic extension of $L^{+}$. Let $A \rightarrow S$ be a principally polarized abelian scheme of relative dimension 16 , with level $n \geq 3$ structure, complex multiplication by $\mathcal{O}_{L}$, and Shimura type $\left(r_{\nu}, s_{\nu}\right)=(0,8),(4,4)$ (for the two embeddings $\nu_{1}, \nu_{2}$ of $F^{+}$in $\mathbb{C}$ ), and level $n$ structure. In the universal case, the base is a Shimura variety of PEL type of dimension $\sum r_{\nu} \cdot s_{\nu}=16$

By functoriality, $\theta_{A}$ commutes with the $\mathcal{O}_{L}$-action, hence respects the decomposition $\mathcal{H}_{d R}^{1}=\oplus_{\rho: L \rightarrow \mathbb{C}} \mathcal{H}_{d R \rho}^{1}$ and the restriction of $\theta_{A, \rho}$ to the summands for $\rho$ above $\nu_{1}$ satisfy $\theta_{A, \rho}=0$ since $r k \Omega_{A} \cap \mathcal{H}_{d R \rho}^{1}=0$ or 8 . Therefore $\max _{\omega} \operatorname{rk} \theta_{A}^{\omega} \leq 8<16$.

In the next three sections, we study instances where the Betti map is of maximal rank, independently of Theorem 2.3.1.

\section{Case Study I: $g \leq 3$.}

In this section, we shall use linear-algebraic arguments to tackle the case $g \leq 3$, proving Theorem 2.3.2 (actually, the case $g=3$ is less elementary and we use a result from [4]). We also notice that Theorem 2.3.2 does not extend to $g=4$ (Remark 7.2.1 (1)).

7.1. We have to show that under the assumptions of Theorem 2.3.2, condition (5.5) holds. We may replace $S$ by a smooth locally closed subvariety of its image under $\mu_{A}$ (in order to reduce to the case $d=d_{\mu_{A}}=g \leq 3$ ), and $A$ by the restriction to $S$ of the universal abelian scheme $\mathcal{X}_{g, n}$. In this situation, for any $s \in S$, the map

$$
T_{S, s} \rightarrow \operatorname{Sym}^{2} \Omega_{A_{s}}^{\vee}, \partial \rightarrow \theta_{A, \partial}(s),
$$

induced by Kodaira-Spencer is injective (cf. [4, 2.1.2]).

7.1.1. Lemma. Suppose $g \leq 3$ and let $W$ be a vector space of dimension $g$ of quadratic forms on $\mathbb{C}^{g}$, containing a non-degenerate quadratic form. There exists a vector on $\mathbb{C}^{g}$ which is not in the kernel of any non-zero quadratic form in $W$.

Proof. We focus on the case $g=3$, leaving to the reader the (easy) verification for $g \leq 2$ (in that case, the assumption that $W$ contains a non-degenerate form is in fact automatic). The content of the above lemma can be rephrased as follows: $\mathbb{P}(W) \subset \mathbb{P}\left(\operatorname{Sym}^{2}\left(\left(\mathbb{C}^{3}\right)^{\vee}\right)\right)$ is a two-dimensional linear system of conics in $\mathbb{P}^{2}$ which, by hypothesis, contains at least one smooth conic. The lemma asserts that there exists a point $p \in \mathbb{P}^{2}$ such that no conic of the linear system $\mathbb{P}(W)$ is singular at $p$. 
Recall that singular conics are either pairs of distinct lines or double lines. In our setting, we start by proving that the set of double lines in $\mathbb{P}(W)$ is finite. In fact, $\mathbb{P}(W)$ is generated by three conics, and if it contains three double lines, these must be in general position (otherwise every conic in $\mathbb{P}(W)$ would contain their intersection and would be singular at that point). But in that case, $W$ is generated by the squares of three independent linear forms, so in suitable coordinates $W$ would consist of the space of diagonal matrices, and $\mathbb{P}(W)$ then contains exactly three double lines.

Thus the set of points which belong to some double line in $\mathbb{P}(W)$ is one-dimensional or empty. Also, since the generic conic in $\mathbb{P}(W)$ is smooth, the set of singular conics in $\mathbb{P}(W)$ is (at most) one-dimensional; each such singular conic either is a double line or has exactly one singular point. Hence the set of points $p \in \mathbb{P}^{2}$ such that $p$ is singular for at least one conic in $\mathbb{P}(W)$ is (at most) one-dimensional, so there is a point outside.

7.2. In order to apply this lemma to (7.1) and derive (5.5), it is enough to show that, under our assumption, at least one $\theta_{A, \partial}$ is of rank $g$, i.e. the Kodaira-Spencer map is non-degenerate in the sense of (5.4).

For $g \leq 2$, this is automatic. For $g=3$, one has the following general theorem of [4], which relies on the theory of automorphic vector bundles: (5.4) holds if $A / S$ is of "restricted PEM type" ( $c f$. [4, 4.2.2]), which means $i$ ) ("PEM") that the connected algebraic monodromy group is maximal with respect to the polarization and the endomorphisms, and $i i$ ) ("restricted") that, in case the center $F$ of $\operatorname{End}_{S} A \otimes \mathbb{Q}$ is a CM field, $\Omega_{A}$ is a free $F \otimes_{\mathbb{Q}} \mathcal{O}_{S}$-module.

It is well-known that all cases with $g=3$ are of PEM type (the first case not of PEM type is the Mumford family with $g=4$ ), and even of restricted PEM type, except when $E n d_{S} A$ is an imaginary quadratic order (over any finite covering of $S$ ). But in this exceptional case, one has $d_{\mu_{A}}=2<g=3[4,4.1 .2]$, which is ruled out by our assumptions.

7.2.1. Remarks. (1) One can follow the pattern of proof of Lemma 7.1.1 for higher values of $g$. This works provided the web $W$ of quadratic forms satisfies the following condition: for each $g^{\prime}=0, \ldots, g$, the algebraic subvariety of $W$ formed of quadratic forms of rank $\leq g^{\prime}$ has dimension $\leq g^{\prime}$. This is not automatic for $g>3$ : for $g=4$, consider the web of quadratic forms spanned by $x_{0}^{2}, x_{0} x_{1}, x_{1}^{2}, x_{2} x_{3}$, which is connected with a fourdimensional family of abelian varieties not satisfying condition (5.5). This family consists of the products $A \times E^{2}$, where $A$ is a principally polarized abelian surface and $E$ an elliptic curve. ${ }^{13}$

(2) The Kodaira-Spencer map of $A / S$ can be described as a trilinear form $\Theta$ on $V_{0} \otimes$ $V_{1} \otimes V_{2}$, where $V_{0}, V_{1}=V_{2}$ are (geometric fibers of) $T_{S}, \Omega_{A}$ respectively - or else, after a choice of basis, as a higher matrix of size $d \times g \times g$. It is symmetric in the last two variables. Conditions (5.4) and (5.5) are non-degeneracy conditions relative to the 1- and 2-slices (resp. 0-slice).

Cayley himself started generalizing his theory of determinants to "trilinear matrices", and the theory of the hyperdeterminant $D e t$ was carried out in full generality by GelfandKapranov-Zelevinsky (if $g \leq d \leq 2 g-1$, Det gives the equation of the dual variety of the Segre variety $\mathbb{P}^{d-1} \times \mathbb{P}^{g-1} \times \mathbb{P}^{g-1}$ ). Let us make precise the connection, referring to the survey [29]. In [29], two further conditions are introduced: "non-degeneracy" and "0non-degeneracy", which in our situation can be translated respectively into the following

\footnotetext{
${ }^{13} \mathrm{Gao}$ has shown [13] that this construction leads to a counter-example to the conclusion of Theorem 2.3.1 if we drop the assumptions.
} 
properties:

$$
\begin{gathered}
\forall \theta, \omega, \eta \neq 0,\left(\theta_{A, \partial}(\omega)=\theta_{A, \partial}(\eta)=0 \Rightarrow \forall \partial^{\prime}, \theta_{A, \partial^{\prime}}(\omega) \cdot \eta=0\right), \\
\forall \omega, \eta \neq 0, \exists \partial, \theta_{A, \partial}(\omega) \cdot \eta \neq 0,
\end{gathered}
$$

the latter being stronger than (5.4). Condition (7.2) is equivalent to Det $\Theta \neq 0$ if $g \leq d \leq$ $2 g-1$, and to (7.3) if $d \geq 2 g-1$ [29, 4.2, 6.1]. The "boundary format" $d=2 g-1$ occurs in the next section.

\section{CASE STUdy II: COMPLEX HYPERELLIPTIC CASE.}

8.1. We treat here the universal hyperelliptic family, where the Kodaira-Spencer map can be made explicit.

Hyperelliptic curves of genus $g \geq 2$ admit a plane model given by an equation of the form $y^{2}=f(x)$, where $f(x) \in \mathbb{C}[x]$ is a polynomial of degree $2 g+1$ without repeated roots. Up to affine transformations on the variable $x$, one can suppose that two of the roots are 0 and 1 ; so the curve is given by

$$
Y_{s}: \quad y^{2}=x(x-1)\left(x-s_{0}\right) \cdots\left(x-s_{2 g-2}\right)=: f(x) ;
$$

our base is

$$
S:=\left\{s=\left(s_{0}, \ldots, s_{2 g-2}\right) \in \mathbb{C}^{2 g-1} \mid s_{i} \neq 0,1, s_{i} \neq s_{j} \text { for } i \neq j\right\} \subset \mathbb{C}^{2 g-1} .
$$

To the curve $Y_{s}$ we associate its jacobian $A_{s}$, thus obtaining a $(2 g-1)$-dimensional family of abelian varieties, over the base $S$. In this case, the map $\mu$ to the moduli space of principally polarized genus $g$ abelian varieties is generically finite.

We identify the tangent space at $Y_{s}$ to the Torelli locus (of jacobians) in $\mathcal{A}_{g}$, with the dual of the vector space of quadratic differentials on $Y_{s}$ (which in turn is isomorphic to $H^{1}\left(Y_{s}, T_{Y_{s}}\right)$ by Serre's duality). A basis for the space $\Gamma\left(Y_{s}, \Omega_{Y_{s}}^{1}\right)$ consists of the 1-forms $\omega_{j}:=\frac{x^{j} \mathrm{~d} x}{y}, j=0, \ldots, g-1$.

The quadratic differentials form a vector space $\Omega_{Y_{s}}^{\otimes 2}$ of dimension $3 g-3$ on which the hyperelliptic involution $\iota$ acts. The $2 g-1$ quadratic differentials $\frac{x^{j} \mathrm{~d} x^{2}}{y^{2}}, j=0, \ldots, 2 g-2$, form a basis of $\iota$-invariants. Note that this space has the same dimension as the moduli space of genus $g$ hyperelliptic curves.

The Kodaira-Spencer map $\theta_{A}$ of $A / S$, in the form of (1.14) in [4], can be viewed, fiberwise, as a linear map $\theta_{A}(s): T_{S}(s) \rightarrow \operatorname{Sym}^{2}\left(\operatorname{Lie} A_{s}\right) \cong \operatorname{Sym}^{2} \Gamma\left(Y_{s}, \Omega_{Y_{s}}\right)^{\vee}$. It is compatible with the Kodaira-Spencer map $\theta_{Y}$ of $Y / S$ in the sense that $\theta_{A}(s)$ is the composition of $\theta_{Y}(s): T_{S}(s) \rightarrow \Gamma\left(Y_{s}, \Omega_{Y_{s}}^{\otimes 2}\right)^{\vee}$ with the transpose of the natural map $\operatorname{Sym}^{2} \Gamma\left(Y_{s}, \Omega_{Y_{s}}\right) \rightarrow \Gamma\left(Y_{s}, \Omega_{Y_{s}}^{\otimes 2}\right)$. The following computation of the Kodaira-Spencer map of $Y / S$ is probably well-known to experts, but we could not find a reference.

8.1.1. Proposition. In the above setting, for each $i=0, \ldots, 2 g-2$, there exists a nonzero number $c_{i}=c_{i}(s)$, depending on the point $s=\left(s_{0}, \ldots, s_{d}\right) \in S$, such that for all $j=0, \ldots, 2 g-2$

$$
\theta_{Y, \partial / \partial s_{i}}\left(\frac{x^{j} \mathrm{~d} x^{2}}{y^{2}}\right)=c_{i} s_{i}{ }^{j} .
$$

Also, for all $j=0, \ldots, g-3, \theta_{Y, \partial / \partial s_{i}}\left(\frac{x^{j} \mathrm{~d} x^{2}}{y}\right)=0$. In particular, the Kodaira-Spencer map induces an isomorphism between the tangent space $T_{S}(s)$ at $s$ and the space of linear functionals on $\Gamma\left(Y_{s}, \Omega_{Y_{s}}^{\otimes 2}\right)$ vanishing on the subspace of odd quadratic differentials. 
This enables to deduce Thm. 2.3.3 from Cor. 2.2.4: since for any $j=0, \ldots, g-1$, multiplication by $\omega_{i, s}$ induces an injection $\Gamma\left(A_{s}, \Omega_{A_{s}}\right) \cong \Gamma\left(Y_{s}, \Omega_{Y_{s}}\right) \rightarrow \Gamma\left(Y_{s}, \Omega_{Y_{s}}^{\otimes 2}\right)_{\text {even }}$, one deduces that formula (5.5) holds with $\omega=\omega_{j}$.

We just outline the strategy of the proof. Given a derivation $\partial$ on the base $S$ (e.g. $\partial=\partial / \partial s_{i}$ ) one can cover the total space of the fibration $\pi: \mathcal{Y} \rightarrow S$ by open sets $U_{\alpha}$ on which there exist derivations $\partial_{\alpha} \in \Gamma\left(U_{\alpha}, T_{\mathcal{Y}}\right)$ such that $\pi_{*}\left(\partial_{\alpha}\right)=\partial$. On $U_{\alpha} \cap U_{\beta} \cap Y_{s}$ the differences $\partial_{\alpha}-\partial_{\beta}$ are derivations on $Y_{s}$ and form a cocyle. One can write $\partial_{\alpha}-\partial_{\beta}$ in $U_{\alpha} \cap U_{\beta}$ as $\tilde{\partial}_{\alpha}-\tilde{\partial}_{\beta}$ for meromorphic derivations $\tilde{\partial}_{\alpha}$ (resp. $\tilde{\partial}_{\beta}$ ) on $U_{\alpha}$ (resp. $U_{\beta}$ ).

Then given a quadratic differential $\omega_{1} \otimes \omega_{2}$ and given a point $P \in Y_{s} \cap U_{\alpha}$ we can calculate the residue at $P$ of the meromorphic 1 -form $\omega_{1}\left(\tilde{\partial}_{\alpha}\right) \cdot \omega_{2}$. The sum over $P \in Y_{s}$ of these numbers equals the value of $\theta_{Y, \partial}\left(\omega_{1} \otimes \omega_{2}\right)$ of the Kodaira-Spencer map at this quadratic differential $\omega_{1} \otimes \omega_{2}$. The outcome of this calculation leads to the formula of Theorem 8.1.1. To be more explicit, let us consider the open set $U_{x}$ in which the $x$-function (as appearing in equation (8.1)) is regular and has non-zero differential and similarly for $U_{y}$. Note that $\left(U_{x} \cup U_{y}\right) \cap Y_{s}$ contains $Y_{s}$ except the unique point at infinity.

Then observe that $\xi:=x^{g} / y$ is a local parameter at infinity. Denote by $U_{\xi}$ a neighborhood of the point at infinity so small so that in $U_{\xi} \backslash\{\infty\}$ the three functions $\xi, x, y$ are regular with non-zero differential.

We then define the derivations $\partial_{i, x}, \partial_{i, y}, \partial_{i, \xi}$ as the unique derivations on the corresponding open sets extending the derivation $\frac{\partial}{\partial s_{i}}$ on $S$ and such that $\partial_{i, u} u=0$ for $u \in\{x, y, \xi\}$. This leads to explicit formulas for $\delta_{x}, \delta_{y}, \delta_{z}$ and explicit choices for $\tilde{\delta}_{x}, \tilde{\delta}_{y}, \tilde{\delta}_{z}$ and an easy calculation of residues gives our formula (8.2).

This shows that for suitable bases of $T_{S}(s)$ and $\Gamma\left(\Omega^{\otimes 2}, Y_{s}\right)^{\vee}$, the matrix of the KodairaSpencer map has the form $(T, 0)$, for a square matrix $T$ of order $2 g-1$, which is a Vandermonde matrix, hence non-singular.

\section{CASE STUDY III: REAL HYPERELLIPTIC CASE.}

In the present section we illustrate a result related to the Betti map for a certain section of the family of Jacobians of hyperelliptic curves of given genus, restricting however to curves and points over $\mathbb{R}$. This leads to issues of a different kind compared to the purely complex case, and we shall present a treatment quite independent of the rest of the paper.

The issues are relevant concerning the paper [32] by R.M. Robinson, as was noted by Serre (see [34]). An independent proof was given recently by B. Lawrence in [20]. Our purpose is to give an alternative argument depending on the Betti map.

9.1. The relevant family of curves and Jacobians. The family we consider is again the hyperelliptic family, but we need to change slightly the notation with respect to the previous section, considering equations of even degree. For $s=\left(s_{0}, \ldots, s_{2 g+1}\right) \in \mathbb{C}^{2 g+2}$, let $f_{s}(x)=x^{2 g+2}+s_{2 g+1} x^{2 g+1}+\ldots+s_{1} x+s_{0}$, let $\Delta(s)$ be its discriminant, and consider the open set $S \subset \mathbb{C}^{2 g+2}$ consisting of the points with $\Delta(s) \neq 0$.

We consider the family $\pi: \mathcal{J} \rightarrow S$ of the Jacobians $J_{s}$, of curves $Y_{s}$ of genus $g$, for $s \in S$, where $Y_{s}$ is affinely defined by the equation

$$
y^{2}=f_{s}(x)
$$

and completed by adding the two poles of the function $x$. We denote them by $\infty^{ \pm}$, where the sign can be specified e.g. by stipulating that $y-x^{g+1}$ has a pole at $\infty^{+}$of order $\leq g$. 
The curve $Y_{s}$ has genus $g=\operatorname{dim} J_{s}$. We consider the section $\sigma$ (of $\pi$ ) on $S$ to $\mathcal{J}$ such that $\sigma(s)=$ class of $\left[\infty^{+}\right]-\left[\infty^{-}\right]$in $J_{s}$, for $s \in S$; it takes real values for every real $s \in S \cap \mathbb{R}^{2 g+2}$. We want to present a proof of the following

9.1.1. Theorem. The set of real $s \in S \cap \mathbb{R}^{2 g+2}$ such that $\sigma(s)$ is a torsion point of $J_{s}$ is dense in $S \cap \mathbb{R}^{2 g+2}$ (for the Euclidean topology).

A main issue here is that we are concerned with real points of the base $S$. For clarity we recall the Betti map for this case.

9.2. Periods, abelian logarithms and the Betti map. There is a locally finite covering of $S$ by open polydisks $U_{\alpha} \subset S, \alpha \in I$, trivializing the holomorphic vector bundle over $S$ given by the tangent spaces to the $J_{s}$, which becomes holomorphically equivalent to $U_{\alpha} \times \mathbb{C}^{g}$ over each $U_{\alpha}$. Through this trivialization, for each $\alpha$ there are column vectors of analytic functions $\omega_{1 \alpha}, \ldots, \omega_{2 g \alpha}: U_{\alpha} \rightarrow \mathbb{C}^{g}$ forming a basis for a lattice $\Lambda_{\alpha, s} \subset \mathbb{C}^{g}$ such that the torus $T_{s}=\mathbb{C}^{g} / \Lambda_{\alpha, s} \cong J_{s}$ analytically (through an exponential map $\exp _{\alpha, s}$ ).

Note that $U_{\alpha} \cap \mathbb{R}^{2 g+2}$ is connected for each $\alpha \in I$ and $U_{\alpha} \cap U_{\beta}$ is connected for any $\alpha, \beta \in I$ (if only because the $U_{\alpha}$ are convex).

9.2.1. Subtori of real points. For real $s \in U_{\alpha} \cap \mathbb{R}^{2 g+2}$, the lattice $\Lambda_{\alpha, s}$ has a sublattice $\Lambda_{\alpha, s}^{\prime}$ of rank $g$, spanning over $\mathbb{R}$ a vector space $V_{\alpha, s}:=\mathbb{R} \Lambda_{\alpha, s}^{\prime}$ of dimension $g$, such that the connected component of the identity in the group $J_{s}(\mathbb{R})$ of real points of $J_{s}$ corresponds to $V_{\alpha, s} / \Lambda_{\alpha, s}^{\prime}$. It is known that $J_{s}(\mathbb{R})$ is a finite union of translates, by torsion points of order 2, of this component; see e.g. Prop. 1.1 in the paper [15] by B. Gross and J. Harris.

It is not difficult to see that we may also choose the above bases so that $\omega_{1 \alpha}(s), \ldots, \omega_{g \alpha}(s)$ span $V_{\alpha, s}$ over $\mathbb{R}$ for $s \in U_{\alpha} \cap \mathbb{R}^{2 g+2}$.

9.2.2. Transition functions. Let $\omega_{\alpha}$ denote the $g \times 2 g$ matrix whose columns are the $\omega_{i \alpha}$. Then on $U_{\alpha} \cap U_{\beta}$ we have a transition formula

$$
\omega_{\beta}=L_{\beta \alpha} \cdot \omega_{\alpha} \cdot R_{\alpha \beta},
$$

where $L_{\beta \alpha} \in G L_{g}\left(\mathcal{O}_{U_{\alpha} \cap U_{\beta}}\right)$ expresses a change of basis of $\mathbb{C}^{g}$, and where $R_{\alpha \beta} \in$ $G L_{2 g}(\mathbb{Z})$ expresses a change of basis for the lattice $\Lambda_{\alpha, s}$.

9.2.3. Abelian logarithms. Since the $U_{\alpha}$ are simply connected, an abelian logarithm $\lambda_{\alpha}$ of $\sigma$ may be defined on each $U_{\alpha}$ as an analytic function to $\mathbb{C}^{g}$. It satisfies $\exp _{\alpha, s} \lambda_{\alpha}(s)=$ $\sigma(s)$. For $s \in U_{\alpha}$, the value $\lambda_{\alpha}(s)$ is uniquely determined up to a vector in $\Lambda_{\alpha, s}=$ $\omega_{\alpha}(s) \mathbb{Z}^{2 g}$. On an intersection $U_{\alpha} \cap U_{\beta}$ as above, such uniqueness and the above transformations (9.2) imply

$$
\lambda_{\beta}=L_{\beta \alpha} \lambda_{\alpha}+\omega_{\beta} v_{\alpha \beta},
$$

for suitable integer column vectors $v_{\alpha \beta} \in \mathbb{Z}^{2 g}$ (constant in $U_{\alpha} \cap U_{\beta}$ ).

9.2.4. Betti coordinates and a restricted Betti map. For $s \in U_{\alpha}$ we may write uniquely

$$
\lambda_{\alpha}(s)=\sum_{i=1}^{2 g} B_{i \alpha}(s) \omega_{i \alpha}(s)=: \omega_{\alpha}(s) B_{\alpha}(s),
$$

for real numbers $B_{i \alpha}(s)$ (and a column vector $B_{\alpha}(s) \in \mathbb{R}^{2 g}$ ). On taking complex conjugates, we see that this gives rise to real functions $B_{i \alpha}$ on $U_{\alpha}$ which are real-analytic in the variable $s \in U_{\alpha} \subset \mathbb{C}^{2 g+2} \cong \mathbb{R}^{4 g+4}$. The above transition transformations (9.2), (9.3) yield, on $U_{\alpha} \cap U_{\beta}$,

$$
B_{\beta}=R_{\alpha \beta}^{-1} B_{\alpha}+v_{\alpha \beta}
$$


By definition we have the (real-analytic) Betti map

$$
B_{\alpha}: U_{\alpha} \rightarrow \mathbb{R}^{2 g}, \quad B_{\alpha}(s)={ }^{t}\left(B_{1, \alpha}(s), \ldots, B_{2 g, \alpha}(s)\right) .
$$

Since for real $s \in A \cap \mathbb{R}^{2 g+2}$ the points $\infty^{ \pm}$lie in $Y_{s}(\mathbb{R})$, the values $\lambda_{\alpha}(s)$ of $\lambda_{\alpha}$ on $U_{\alpha} \cap \mathbb{R}^{2 g+2}$ lie, modulo $\Lambda_{\alpha, s}$, in one of the above mentioned finitely many translates of $V_{\alpha, s}$. Now, recalling that the translates in question are obtained by torsion points of order 2 , this yields that $B_{\alpha}\left(U_{\alpha} \cap \mathbb{R}^{2 g+2}\right)$ is inside $\mathbb{R}^{g} \times\{0\}+\frac{1}{2} \mathbb{Z}^{2 g}$, due to the present choice of $\omega_{1 \alpha}, \ldots, \omega_{g \alpha}$. By continuity and connectedness, the last $g$ coordinates of the map are constant on $U_{\alpha} \cap \mathbb{R}^{2 g+2}$.

Then let us now denote by $B_{\mathbb{R} \alpha}: U_{\alpha} \cap \mathbb{R}^{2 g+2} \rightarrow \mathbb{R}^{g}$ the projection to the first $g$ coordinates of the map $B_{\alpha}$ restricted to real points; this is still real-analytic. This really is meaningful only if the relevant set is non-empty, so, also for later reference, we define $I_{0} \subset I$ as the subset of the $\alpha \in I$ such that $U_{\alpha} \cap \mathbb{R}^{2 g+2}$ is non-empty.

9.3. The differential of the restricted Betti map. Let $s \in U_{\alpha}$; then the value $\sigma(s)$ is torsion on $J_{s}$ if and only if $B_{\alpha}(s) \in \mathbb{Q}^{2 g}$. Then, to prove Theorem 9.1.1 it will suffice to prove

9.3.1. Theorem. For each $\alpha \in I_{0}$ the map $B_{\mathbb{R} \alpha}$ on $U_{\alpha} \cap \mathbb{R}^{2 g+2}$ attains rational values on a dense subset of $U_{\alpha} \cap \mathbb{R}^{2 g+2}$.

For this, the first thing we want to show is that its differential has maximal rank $g$ at some real point $s \in U_{\alpha} \cap \mathbb{R}^{2 g+2}$ in case this set is non-empty, and in turn we shall prove this by comparison with the dimension of the fibers. We start with:

9.3.2. Proposition. Suppose that for a certain $\alpha \in I_{0}$ and every point $s \in U_{\alpha} \cap \mathbb{R}^{2 g+2}$ the differential of $B_{\mathbb{R} \alpha}$ has rank $<g$ at $s$. Then, for all points $u \in U_{\alpha} \cap \mathbb{R}^{2 g+2}$ the fiber $B_{\alpha}^{-1}\left(B_{\alpha}(u)\right) \subset U_{\alpha}$ is a complex variety in $U_{\alpha}$ of complex dimension $>g+2$.

Proof. The assumption implies, through the (real) implicit function theorem, that at all points $u$ in an open dense subset of $U_{\alpha} \cap \mathbb{R}^{2 g+2}$ the fiber $B_{\mathbb{R} \alpha}^{-1}\left(B_{\mathbb{R} \alpha}(u)\right)$ of $B_{\mathbb{R} \alpha}$ on $U_{\alpha} \cap$ $\mathbb{R}^{2 g+2}$ has real dimension $>g+2$. So, the complex fiber $B_{\alpha}^{-1}\left(B_{\alpha}(u)\right) \subset U_{\alpha}$ at such real points $u$ has a real subset (that is, a subset inside $U_{\alpha} \cap \mathbb{R}^{2 g+2}$ ) of real dimension $>g+2$, since this complex fiber contains the respective fiber $B_{\mathbb{R} \alpha}^{-1}\left(B_{\mathbb{R} \alpha}(u)\right) \subset U_{\alpha} \cap \mathbb{R}^{2 g+2}$.

But the fibers of $B_{\alpha}$ (on $U_{\alpha}$ ) are complex varieties inside $U_{\alpha}$; in fact, the fiber $B_{\alpha}^{-1}\left(b_{1}, \ldots, b_{2 g}\right)$ is defined in $U_{\alpha}$ by the complex-analytic equation $\lambda_{\alpha}(z)=$ $\sum_{i=1}^{2 g} b_{i} \omega_{i \alpha}(z)$. We deduce that the complex dimension at $u$ of the fiber $B_{\alpha}^{-1}\left(B_{\alpha}(u)\right)$ (as a subset of $U_{\alpha}$ ) is $>g+2$ for all $u$ in a suitable open dense subset of $U_{\alpha} \cap \mathbb{R}^{2 g+2}$.

The sought conclusion of the Proposition now follows immediately from the following result, used also in the recent paper [7], to which we refer for a proof:

For every $k \in \mathbb{N}$, the set $\left\{s \in U_{\alpha}: \operatorname{dim}_{s} B_{\alpha}^{-1}\left(B_{\alpha}(s)\right) \geq k\right\}$ is closed in $U_{\alpha}$.

Next, we want to extend the conclusion of the proposition (keeping the same assumption) to a whole connected component of $S \cap \mathbb{R}^{2 g+2}$ :

9.3.3. Proposition. Suppose that for some $\alpha \in I_{0}$ and every point $s \in U_{\alpha} \cap \mathbb{R}^{2 g+2}$ the differential of $B_{\mathbb{R} \alpha}$ has rank $<g$ at $s$. Then for every $u \in A \cap \mathbb{R}^{2 g+2}$ lying in the same connected component of $U_{\alpha} \cap \mathbb{R}^{2 g+2}$, and for every $\gamma$ such that $u \in U_{\gamma}$, the fiber $B_{\gamma}^{-1}\left(B_{\gamma}(u)\right) \subset S$ has complex dimension $>g+2$ at $u$.

Proof. Let $I_{0}^{\prime}$ be the subset of indices $\beta \in I_{0}$ such that the differential of $B_{\mathbb{R} \beta}$ has rank $<g$ everywhere on $U_{\beta} \cap \mathbb{R}^{2 g+2}$. We contend that the union $\bigcup_{\beta \in I_{0}^{\prime}}\left(U_{\beta} \cap \mathbb{R}^{2 g+2}\right)$ is open and closed in $A \cap \mathbb{R}^{2 g+2}$, hence a union of connected components of $S \cap \mathbb{R}^{2 g+2}$. 
Now, this union is open since every $U_{\alpha}$ is open. To prove it is closed in $S \cap \mathbb{R}^{2 g+2}$, let $\gamma \in I_{0}^{\prime}$ and let $\delta \in I_{0}$ be such that $U_{\gamma} \cap U_{\delta} \cap \mathbb{R}^{2 g+2}$ is non-empty.

Note that the transformation (9.5) (and our convention about the choice and orderings of the bases $\omega_{\gamma}, \omega_{\delta}$ ) yields that $B_{\mathbb{R} \delta}=\Gamma_{\gamma \delta} B_{\mathbb{R} \gamma}+\frac{1}{2} \tilde{v}_{\gamma \delta}$ on $U_{\gamma} \cap U_{\delta} \cap \mathbb{R}^{2 g+2}$, for a suitable integral $g \times g$ matrix $\Gamma_{\gamma \delta}$ and a suitable $\tilde{v}_{\gamma \delta} \in \mathbb{Z}^{g}$.

Then the differential of $B_{\mathbb{R} \delta}$ shall have rank $<g$ on $U_{\gamma} \cap U_{\delta} \cap \mathbb{R}^{2 g+2}$, hence on the whole $U_{\delta} \cap \mathbb{R}^{2 g+2}$, for the former set is non-empty and open in the latter, the latter is connected and the relevant map is real-analytic. In other words, $\delta \in I_{0}^{\prime}$ too.

The closure of $\bigcup_{\beta \in I_{0}^{\prime}}\left(U_{\beta} \cap \mathbb{R}^{2 g+2}\right)$ follows: let $s \in A \cap \mathbb{R}^{2 g+2}$ lie in the complement (with respect to $S \cap \mathbb{R}^{2 g+2}$ ); this $s$ lies in some $U_{\eta} \cap \mathbb{R}^{2 g+2}$ and then, by what we have seen, $U_{\eta} \cap \mathbb{R}^{2 g+2}$ shall be disjoint from our set. Therefore the complement of our set is open, as asserted.

It follows that if $\alpha \in I_{0}^{\prime}$, then $\gamma \in I_{0}^{\prime}$ for every $\gamma \in I_{0}$ such that $U_{\gamma} \cap \mathbb{R}^{2 g+2}$ is in the same connected component of $U_{\alpha} \cap \mathbb{R}^{2 g+2}$ (relative to $S \cap \mathbb{R}^{2 g+2}$ ).

Now, Proposition 9.3.3 follows on applying Proposition 9.3.2 to each such $\gamma$.

9.4. Discussion around Proposition 9.3.3. To contradict the conclusion of this proposition, hence proving that its assumption cannot hold, let us first inspect the connected components of $S \cap \mathbb{R}^{2 g+2}$. Each point $s \in A \cap \mathbb{R}^{2 g+2}$ corresponds to a real monic polynomial of degree $2 g+2$ with no multiple complex root. Let $2 r(s)$ be the number of real roots, so $r(s)$ is an integer, $0 \leq r(s) \leq g+1$. It is easy to see that $r(s)$ is locally constant in $S \cap \mathbb{R}^{2 g+2}$ and then it readily follows that each (non-empty) connected component is defined in $S \cap \mathbb{R}^{2 g+2}$ by an equation $r(s)=r$, where $r$ is a given integer in $[0, g+1]$.

We have the following elementary lemma, whose proof we leave to the interested reader:

9.4.1. Lemma. For each $r \in\{0,1, \ldots, g+1\}$ there exist a monic polynomial $P \in \mathbb{R}[x]$ of degree $g+1$ and a real number $p \neq 0$ such that $P(x)^{2}-p$ has precisely $2 r$ simple real roots and no multiple complex root.

Let now $\alpha \in I_{0}$ be such that the assumption of Proposition 9.3.3 holds for $U_{\alpha} \cap \mathbb{R}^{2 g+2}$, and let $K$ be the connected component of $U_{\alpha} \cap \mathbb{R}^{2 g+2}$ in $S \cap \mathbb{R}^{2 g+2}$. In the above description, $K$ corresponds to an integer $r \in[0, g+1]$. Let us apply the lemma to it and let $P(x), p$ the polynomial and real number coming from the lemma. Then the polynomial $P(x)^{2}-p$ corresponds, in our opening notation, to a point $s \in K$, so that $f_{s}(x)=P(x)^{2}-p$.

The identity $P(x)^{2}-f_{s}(x)=p \neq 0$ shows that the function $P(x)+y$ on $Y_{s}$ has divisor $(g+1)\left(\left[\infty^{+}\right]-\left[\infty^{-}\right]\right)$, hence $\sigma(s)$ is torsion in $J_{s}$, of order (dividing but in fact equal to) $g+1$. Thus, for every $\gamma$ with $s \in U_{\gamma}$, the point $\rho:=B_{\gamma}(s)$ is a rational point in $\mathbb{Q}^{2 g}$ with denominator dividing $g+1$.

Let us look at the complex fiber $B_{\gamma}^{-1}(\rho)$ around $s$ : it consists of complex points $t \in S$ such that $\sigma(t)$ has torsion order dividing $g+1$ on the Jacobian $J_{t}$ of $Y_{t}$. Hence for such a $t$ there is a rational function on $Y_{t}$ with divisor $(g+1)\left(\left[\infty^{+}\right]-\left[\infty^{-}\right]\right)$; this corresponds to a polynomial $P_{t}$ of degree $g+1$ such that $P_{t}(x)^{2}=f_{t}(x)+p_{t}$ for some nonzero complex number $p_{t}$. This $P_{t}(x)$ is monic of degree $g+1$, hence it depends on $\leq g+1$ complex parameters; taking also $p_{t}$ into account, we see that the complex dimension of the said fiber cannot be $>g+2$, yielding the sought contradiction.

9.5. Conclusion of the argument. We have thus proved that for every $\alpha \in I_{0}$ there exists some point $s \in U_{\alpha} \cap \mathbb{R}^{2 g+2}$ such that the differential of $B_{\mathbb{R} \alpha}$ has rank $\geq g$, hence maximal rank $g$, at $s$. Then such differential has maximal rank on a dense open subset $U_{\alpha}^{\prime}$ of (real) points in $U_{\alpha} \cap \mathbb{R}^{2 g+2}$. At this stage we can invoke the following general fact: 
Let $f: X \rightarrow Y$ be a continuous map between topological spaces; suppose there is an open dense subset $X^{\prime}$ of $X$ such that $f_{\mid X^{\prime}}: X^{\prime} \rightarrow Y$ is open. Let $Z$ be a dense subset of $Y$. Then $f^{-1}(Z)$ is dense in $X$.

Proof. Suppose not. Then, there is a non-empty open subset $V$ of $X$ such that $f(V) \cap Z$ is empty. Since $X^{\prime}$ is dense, $X^{\prime} \cap V$ is not empty. Because $f_{\mid X^{\prime}}$ is open, $f\left(X^{\prime} \cap V\right)$ is open in $Y$ and does not intersect $Z$ : this contradicts the fact that $Z$ is dense in $Y$.

Now we may apply this statement to $X=U_{\alpha} \cap \mathbb{R}^{2 g+2}, f=B_{\mathbb{R} \alpha}$ and to $X^{\prime}=U_{\alpha}^{\prime}$, taking for $Z$ the set of rational points in $B_{\mathbb{R} \alpha}(X)$. Theorem 9.3.1 follows.

Remark. One might ask about a $p$-adic analogue; it seems to us that a density statement does not hold.

\section{Appendix by Z. GaO: an APPliCation of the pure AX-SChanUel \\ THEOREM.}

10.1. Main Result. Let $\mathcal{A}_{g}$ be the moduli space of principally polarized abelian varieties of dimension $g$, possibly with some level structure.

Let $S$ be a complex irreducible algebraic variety. Let $\pi: A \rightarrow S$ be an abelian scheme of relative dimension $g$. We may assume that $A / S$ is principally polarized up to replacing $A$ by an isogeneous abelian scheme. Then $\pi: A \rightarrow S$ induces a modular map $\mu_{A}: S \rightarrow \mathcal{A}_{g}$. We assume $\operatorname{dim} \mu_{A}(S) \geq g$.

Next we want to understand when $S$ satisfies the conclusion of Cor. 2.2.2. More precisely, let $\mathfrak{H}_{g}$ be the Siegel upper half space and let $u: \mathfrak{H}_{g} \rightarrow \mathcal{A}_{g}$ be the uniformization. Denote by $\tilde{S}$ a complex analytic irreducible component of $u^{-1}\left(\mu_{A}(S)\right)$ in $\mathfrak{H}_{g}$. We name

Condition $A C Z$ For any $\tilde{s} \in \tilde{S}$ and any $\mathbf{c} \in \mathbb{C}^{g}$, there exists a complex analytic subvariety $\tilde{C} \subset \tilde{U}$ of dimension $\operatorname{dim} \mu_{A}(S)-g+1$ passing through $\tilde{s} \in \mathfrak{H}_{g}$ such that $\tilde{s}^{\prime} \mathbf{c}$ is constant for any $\tilde{s}^{\prime} \in \tilde{C}$. Here we view $\mathbf{c} \in \mathbb{C}^{g}$ as a column vector and $\tilde{s}^{\prime} \mathbf{c}$ is the usual matrix product (recall that every point in $\mathfrak{H}_{g}$ is a $g \times g$ matrix).

Our main result is:

10.1.1. Theorem. If Condition ACZ is satisfied, then $\mu_{A}(S)$ is contained in a proper special subvariety of $\mathcal{A}_{g}$.

10.1.2. Remark. There are several equivalent ways to state the conclusion of Theorem 10.1.1. In fact for any irreducible subvariety $S$ of $\mathcal{A}_{g}$, we shall prove in Lemma 10.2.6 that the following statements are equivalent:

(i) The variety $S$ is not contained in any proper special subvariety of $\mathcal{A}_{g}$.

(ii) The variety $S$ contains a point with Mumford-Tate group $G S p_{2 g}$.

(iii) The monodromy group of $A / S$ is Zariski dense in $S p_{2 g}$.

In practice, condition (i) is often checked by computation of the Mumford-Tate group (hence condition (ii)) or of the monodromy group (hence condition (iii)). For example as $A / S$ is not isotrivial by Assumption 1, if we denote by $V=H^{1}\left(A_{s}, \mathbb{C}\right)$ for any $s \in S$, then condition (iii) holds if (and only if) the symmetric square $S^{2} V$ is irreducible for the monodromy action by Beukers-Brownawell-Heckman [8, Theorem 2.2].

In fact, Theorem 10.1.1 can be deduced from a more technical and more general theorem. In order to state the theorem we need to introduce some notation.

Recall the uniformization $u: \mathfrak{H}_{g} \rightarrow \mathcal{A}_{g}$. The natural embedding $\mathfrak{H}_{g} \subset \mathbb{C}^{g(g+1) / 2}$ endows $\mathfrak{H}_{g}$ with a structure of "complex algebraic variety", and hence $u$ gives rise to a bi-algebraic system. Say a complex analytic irreducible subset $\tilde{Y}$ of $\mathfrak{H}_{g}$ is bi-algebraic if $\tilde{Y}$ is algebraic in $\mathfrak{H}_{g}$ and $u(\tilde{Y})$ is algebraic in $\mathcal{A}_{g}$. See $\S 10.2$ for more details. 
Denote by $H_{S}^{\circ}$ the connected algebraic monodromy group of $A / S$, namely $H_{S}^{\circ}$ is the neutral component of the Zariski closure of $\operatorname{im}\left(\pi_{1}(S, s) \rightarrow \pi_{1}\left(\mathcal{A}_{g}, s\right)\right) \subset S p_{2 g}(\mathbb{Z})$ in $S p_{2 g}$. Denote by $\tilde{S}^{\text {biZar }}$ the smallest bi-algebraic subset of $\mathfrak{H}_{g}$ which contains $\tilde{S}$. It exists by Lemma 10.2.3.

Recall that every element of $\mathfrak{H}_{g}$ is a $g \times g$-matrix. For any $\mathbf{c} \in \mathbb{C}^{g}$ and any $\tilde{s} \in \mathfrak{H}_{g}$, denote by

$$
H_{\mathbf{c}, \tilde{s}}:=\left\{Z \in \mathfrak{H}_{g}: Z \mathbf{c}=\tilde{s} \mathbf{c}\right\} .
$$

10.1.3. Theorem. There does not exist an abelian scheme $A / S$ with $\operatorname{dim} \mu_{A}(S) \geq g$ satisfying the following three properties:

(i) The connected algebraic monodromy group $H_{S}^{\circ}$ is simple;

(ii) There exist $\mathbf{c} \in \mathbb{C}^{g}$ and $\tilde{s} \in \tilde{S}$ such that $\operatorname{codim}_{\tilde{S}}$ biZar $\left(H_{\mathbf{c}, \tilde{s}} \cap \tilde{S}^{\text {biZar }}\right)=g$.

(iii) Condition ACZ is satisfied.

10.2. Review on the bi-algebraic system of $\mathcal{A}_{g}$ and Ax-Schanuel. We focus on the case $\mathcal{A}_{g}$. We shall consider the uniformization $u: \mathfrak{H}_{g} \rightarrow \mathcal{A}_{g}$, where $\mathfrak{H}_{g}$ is the Siegel upper half space defined as the following.

$$
\mathfrak{H}_{g}=\left\{Z=X+\sqrt{-1} Y \in \operatorname{Mat}_{g \times g}(\mathbb{C}): Z=Z^{\mathrm{t}}, Y>0\right\} .
$$

Later on we will study subvarieties of $\mathfrak{H}_{g}$ and $\mathcal{A}_{g}$ at the same time. To distinguish them we often use letters to denote subsets of $\mathcal{A}_{g}$ and add a $\sim$ on top to denote subsets of $\mathfrak{H}_{g}$.

Consider

$$
\mathfrak{p}_{g}=\left\{Z \in \operatorname{Mat}_{g \times g}(\mathbb{C}): Z=Z^{\mathrm{t}}\right\} .
$$

Then $\mathfrak{p}_{g} \cong \mathbb{C}^{g(g+1) / 2}$ as $\mathbb{C}$-vector spaces. The Siegel upper half space is open (in the usual topology) and semi-algebraic in $\mathfrak{p}_{g}$. The complex structure on $\mathfrak{p}_{g}$ thus induces a structure of complex analytic variety on $\mathfrak{H}_{g}$. Following Pila, Ullmo and Yafaev, we define

10.2.1. Definition. A subset $\tilde{Y}$ of $\mathfrak{H}_{g}$ is said to be irreducible algebraic if it is a complex analytic irreducible component of $\mathfrak{H}_{g} \cap \tilde{Y}^{\mathrm{c}}$ for some algebraic subvariety $\tilde{Y}^{\mathrm{c}}$ of $\mathfrak{p}_{g}$.

Hence we have the following definition.

10.2.2. Definition. (1) A subset $\tilde{F}$ of $\mathfrak{H}_{g}$ is said to be bi-algebraic if it is irreducible algebraic in $\mathfrak{H}_{g}$ and $u(\tilde{F})$ is an algebraic subvariety of $\mathcal{A}_{g}$.

(2) An irreducible subvariety $F$ of $\mathcal{A}_{g}$ is said to be bi-algebraic if one (and hence any) complex analytic irreducible component of $u^{-1}(F)$ is irreducible algebraic in $\mathfrak{H}_{g}$.

Before moving on, let us make the following observation.

10.2.3. Lemma. Let $F_{1}$ and $F_{2}$ be two bi-algebraic subvarieties of $\mathcal{A}_{g}$, and let $F$ be an irreducible component of $F_{1} \cap F_{2}$. Then $F$ is also bi-algebraic.

Proof. First $F$ is clearly irreducible algebraic. Consider a complex analytic irreducible component $\tilde{F}$ of $u^{-1}(F)$. It is contained in both $\tilde{F}_{1}$, an irreducible component of $u^{-1}\left(F_{1}\right)$, and $\tilde{F}_{2}$, an irreducible component of $u^{-1}\left(F_{2}\right)$. Let $\tilde{F}^{\prime}$ be an irreducible component of $\tilde{F}_{1} \cap \tilde{F}_{2}$ which contains $\tilde{F}$. Then $F=u(\tilde{F})=u\left(\tilde{F}^{\prime}\right) \subset u\left(\tilde{F}_{1}\right) \cap u\left(\tilde{F}_{2}\right)=F_{1} \cap F_{2}$. Taking the Zariski closures, we get $F \subset u\left(\tilde{F}^{\prime}\right)^{Z a r} \subset F_{1} \cap F_{2}$. Now since $\tilde{F}^{\prime}$ is irreducible, we know that $u\left(\tilde{F}^{\prime}\right)^{Z a r}$ is irreducible. Hence $F=u\left(\tilde{F}^{\prime}\right)^{Z a r}$ since $F$ is an irreducible component of $F_{1} \cap F_{2}$. Therefore $F=u\left(\tilde{F}^{\prime}\right)$ and so $\tilde{F}=\tilde{F}^{\prime}$ is algebraic. So $F$ is bi-algebraic. 
Based on this observation, for any irreducible subvariety $Y$ of $\mathcal{A}_{g}$, there exists a unique smallest bi-algebraic subvariety of $\mathcal{A}_{g}$ which contains $Y$. Then for any complex analytic irreducible subset $\tilde{Y}$ of $\mathfrak{H}_{g}$, there exists a unique smallest bi-algebraic subset of $\mathfrak{H}_{g}$ which contains $\tilde{Y}$ : it is an irreducible component of the smallest bi-algebraic subvariety of $\mathcal{A}_{g}$ which contains $u(\tilde{Y})^{Z a r}$.

There is a better characterization of bi-algebraic subvarieties of $\mathcal{A}_{g}$ using Hodge theory and group theory. They are precisely the so-called weakly special subvarieties of $\mathcal{A}_{g}$ defined by Pink [30, Definition 4.1.(b)]. This is proven by Ullmo-Yafaev [35, Theorem 1.2]. Moonen has also studied these subvarieties and proved that they are precisely the totally geodesic subvarieties of $\mathcal{A}_{g}$. See [26, 4.3]. Linearity properties in Shimura varieties was first studied by Moonen in loc.cit. For our purpose we prove the following lemma.

10.2.4. Lemma. Let $\tilde{F}$ be a bi-algebraic subset of $\mathfrak{H}_{g}$. Then it is affine linear, meaning that it is the intersection of $\mathfrak{H}_{g}$ with some affine linear subspace of $\mathfrak{p}_{g}$.

Proof. This follows from Ullmo-Yafaev's characterization and the Harish-Chandra realization of Hermitian symmetric domains. Let us explain the details. We use the language of Shimura data in the proof.

By a result of Ullmo-Yafaev [35, Theorem 1.2], bi-algebraic subsets of $\mathfrak{H}_{g}$ are precisely the weakly special subsets of $\mathfrak{H}_{g}$. Hence $\tilde{F}$ is a weakly special subset of $\mathfrak{H}_{g}$. By definition of weakly special subvarieties (see [35, Definition 2.1] or [30, Definition 4.1.(b)]), there exist a connected Shimura subdatum $(G, \mathcal{X})$ of $\left(G S p_{2 g}, \mathfrak{H}_{g}\right)$ and a decomposition $\left(G^{a d}, \mathcal{X}\right)=\left(G_{1}, \mathcal{X}_{1}\right) \times\left(G_{2}, \mathcal{X}_{2}\right)$ and a point $\tilde{x}_{2} \in \mathcal{X}_{2}$ such that $\tilde{F}=\mathcal{X}_{1} \times\left\{\tilde{x}_{2}\right\}$.

Take any point $\left(\tilde{x}_{1}, \tilde{x}_{2}\right) \in \mathcal{X}_{1} \times\left\{\tilde{x}_{2}\right\} \subset \mathcal{X}$, we have the Harish-Chandra embedding of $\mathcal{X}$ into $T_{\left(\tilde{x}_{1}, \tilde{x}_{2}\right)} \mathcal{X}$, the tangent space of $\mathcal{X}$ at $\left(\tilde{x}_{1}, \tilde{x}_{2}\right)$. We have also the Harish-Chandra embedding of $\mathfrak{H}_{g}$ into $T_{\left(\tilde{x}_{1}, \tilde{x}_{2}\right)} \mathfrak{H}_{g}$. These two embeddings are compatible in the following sense: $T_{\left(\tilde{x}_{1}, \tilde{x}_{2}\right)} \mathcal{X}$ is a linear subspace of $T_{\left(\tilde{x}_{1}, \tilde{x}_{2}\right)} \mathfrak{H}_{g}$ and $\mathcal{X}=\mathfrak{H}_{g} \cap T_{\left(\tilde{x}_{1}, \tilde{x}_{2}\right)} \mathcal{X}$. This is proven in Helgason [16, Chapter VIII, §7]. We refer to [24, Chapter 5, §2, Theorem 1] for the presentation. In particular, the Harish-Chandra embedding realizes $\mathfrak{H}_{g}$ as the unit ball in $\mathbb{C}^{g(g+1) / 2}$.

The natural embedding of the Hermitian symmetric space $\mathfrak{H}_{g}$ into $\mathfrak{p}_{g}$ can be realized as the Harish-Chandra embedding mentioned above composed with a linear transformation which we call $\ell$. Define $\mathfrak{p}=\ell\left(T_{\left(\tilde{x}_{1}, \tilde{x}_{2}\right)} \mathcal{X}\right)$. Then $\mathfrak{p}$ is an affine subspace of $\mathfrak{p}_{g}$. Now by the compatibility mentioned in the last paragraph, we have that $\mathcal{X}=\mathfrak{p} \cap \mathfrak{H}_{g}$.

The decomposition of Hermitian symmetric spaces $\mathcal{X}=\mathcal{X}_{1} \times \mathcal{X}_{2}$ gives a decomposition $\mathfrak{p}=\mathfrak{p}_{1} \times \mathfrak{p}_{2}$ as complex spaces, and $\tilde{F}=\mathcal{X}_{1} \times\left\{\tilde{x}_{2}\right\}$ is then $\left(\mathfrak{p}_{1} \times\{0\}\right) \cap \mathcal{X}=\left(\mathfrak{p}_{1} \times\right.$ $\{0\}) \cap \mathfrak{H}_{g}$. Hence we are done.

Now we are ready to state the Ax-Schanuel theorem for $\mathcal{A}_{g}$. It is recently proven by Mok-Pila-Tsimerman [25]. This theorem has several equivalent forms, whose equivalences are not hard to show. For our purpose we only need the following weak form.

10.2.5. Theorem. Let $\tilde{Y}$ be an irreducible complex analytic subvariety of $\mathfrak{H}_{g}$. Let $\tilde{Y}^{\text {biZar }}$ be the smallest bi-algebraic subset of $\mathfrak{H}_{g}$ which contains $\tilde{Y}$. Then

$$
\operatorname{dim} \tilde{Y}^{Z a r}+\operatorname{dim} u(\tilde{Y})^{Z a r} \geq \operatorname{dim} \tilde{Y}+\operatorname{dim} \tilde{Y}^{\text {biZar }} .
$$

Here $\tilde{Y}^{Z a r}$ means the smallest irreducible algebraic subset of $\mathfrak{H}_{g}$ which contains $\tilde{Y}$.

We end this section by proving the equivalence of the following statements. 
10.2.6. Lemma. Let $S$ be an irreducible subvariety of $\mathcal{A}_{g}$. Then the following statements are equivalent:

(i) The variety $S$ is not contained in any proper special subvariety of $\mathcal{A}_{g}$.

(ii) The variety $S$ contains a point with Mumford-Tate group $G S p_{2 g}$.

(iii) The monodromy group of $A / S$ is Zariski dense in $S p_{2 g}$.

(iv) The variety $S$ is not contained in any proper bi-algebraic subvariety of $\mathcal{A}_{g}$ of positive dimension.

(v) There exists a point $s \in S(\mathbb{C})$ such that the following condition holds: $s$ is not contained in any proper bi-algebraic subvariety of $\mathcal{A}_{g}$ of positive dimension.

Proof. By Deligne-André, a very general point in $S(\mathbb{C})$ has the same Mumford-Tate group which we denote by $\operatorname{MT}(S)$. The Mumford-Tate group $\operatorname{MT}(S)$ is a reductive group, and a subgroup of finite index of the monodromy group is contained in $\operatorname{MT}(S)^{\text {der }}$. Here "very general" means that the point is taken outside an at most countable union of proper subvarieties of $S$. We refer to [2, Lemma 4] for these facts.

Now let us prove (iii) $\Rightarrow$ (i). If the monodromy group of $A / S$ is Zariski dense in $S p_{2 g}$, then $S p_{2 g}$ is a subgroup of $\mathrm{MT}(S)^{\mathrm{der}}<G S p_{2 g}^{\mathrm{der}}=S p_{2 g}$. Hence $\mathrm{MT}(S)^{\mathrm{der}}=S p_{2 g}$. So $\operatorname{MT}(S)=G S p_{2 g} \cdot{ }^{14}$

For (ii) $\Rightarrow$ (iii), we use a stronger result of André. Let $H_{S}^{\circ}$ be the neutral component of the Zariski closure of the monodromy group of $A / S$ in $G S p_{2 g}$. Then by [2, Theorem 1], we have that $H_{S}^{\circ}$ is a non-trivial normal subgroup of $G S p_{2 g}^{\mathrm{der}}=S p_{2 g}$. But $S p_{2 g}$ is simple, so $H_{S}^{\circ}=S p_{2 g}$.

Let us prove (i) $\Rightarrow$ (ii). The smallest special subvariety of $\mathcal{A}_{g}$ which contains $S$ is defined by a Shimura subdatum with underlying group $\mathrm{MT}(S)$. If $\mathrm{MT}(S) \neq G S p_{2 g}$, then the smallest special subvariety of $\mathcal{A}_{g}$ is not $\mathcal{A}_{g}$, which contradicts the assumption of (i).

We have (iv) $\Rightarrow$ (i) since every special subvariety of $\mathcal{A}_{g}$ is bi-algebraic.

The implication $(\mathrm{v}) \Rightarrow$ (iv) is easy.

It remains to prove (ii) $\Rightarrow(\mathrm{v})$. Let $s \in S(\mathbb{C})$ be such that $\mathrm{MT}(s)=G S p_{2 g}$. Take $\tilde{s} \in$ $u^{-1}(s)$. Let $F$ be a bi-algebraic subvariety of $\mathcal{A}_{g}$ which contains $s$ with $\operatorname{dim} F>0$. Let $\tilde{F}$ be an irreducible component of $u^{-1}(F)$ which contains $\tilde{s}$. It suffices to prove $\tilde{F}=\mathfrak{H}_{g}$.

The proof goes as follows. By a result of Ullmo-Yafaev [35, Theorem 1.2], bi-algebraic subsets of $\mathfrak{H}_{g}$ are precisely the weakly special subsets of $\mathfrak{H}_{g}$. Hence $\tilde{F}$ is a weakly special subset of $\mathfrak{H}_{g}$. By definition of weakly special subvarieties (see [35, Definition 2.1] or [30, Definition 4.1.(b)]), there exist a connected Shimura subdatum $(G, \mathcal{X})$ of $\left(G S p_{2 g}, \mathfrak{H}_{g}\right)$ and a decomposition $\left(G^{\text {ad }}, \mathcal{X}\right)=\left(G_{1}, \mathcal{X}_{1}\right) \times\left(G_{2}, \mathcal{X}_{2}\right)$ and a point $\tilde{x}_{2} \in \mathcal{X}_{2}$ such that $\tilde{F}=\mathcal{X}_{1} \times\left\{\tilde{x}_{2}\right\}$. The condition $\operatorname{MT}(\tilde{s})=G S p_{2 g}$ implies that the smallest Shimura subdatum of $\left(G S p_{2 g}, \mathfrak{H}_{g}\right)$ whose underlying space contains $\tilde{s}$ is $\left(G S p_{2 g}, \mathfrak{H}_{g}\right)$. Therefore $(G, \mathcal{X})=\left(G S p_{2 g}, \mathfrak{H}_{g}\right)$. But then $G^{\text {ad }}=G S p_{2 g}^{\text {ad }}$ is a simple group, and hence either $\tilde{F}=\mathfrak{H}_{g}$ or $\tilde{F}$ is a point. But $\operatorname{dim} \tilde{F}>0$, so $\tilde{F}=\mathfrak{H}_{g}$.

10.3. Proof of Theorem 10.1.1. We may replace $S$ by $\mu_{A}(S)$ and hence assume that $S$ is an irreducible subvariety of $\mathcal{A}_{g}$ of dimension $\geq g$. Recall the uniformization $u: \mathfrak{H}_{g} \rightarrow \mathcal{A}_{g}$ and our convention that $\tilde{S}$ is a complex analytic irreducible component of $u^{-1}(S)$.

The key to prove Theorem 10.1.1 is the following proposition, whose proof uses AxSchanuel.

\footnotetext{
${ }^{14}$ By Hodge theory, $\mathbb{G}_{m}=Z\left(G S p_{2 g}\right)$ is contained in $\mathrm{MT}(S)$. In this paper we only need $S p_{2 g}<\mathrm{MT}(S)$.
} 
10.3.1. Proposition. Suppose Condition ACZ is satisfied. Then for any $\tilde{s} \in \tilde{S}$, there exists a bi-algebraic subset $\tilde{F}$ of positive dimension, properly contained in $\mathfrak{H}_{g}$, such that $\tilde{s} \in \tilde{F}$.

Proof. Fix a $\mathbf{c} \in \mathbb{C}^{g}$ and define the following subspace of $\mathfrak{H}_{g}$

$$
H_{\mathbf{c}, \tilde{s}}:=\left\{Z \in \mathfrak{H}_{g}: Z \mathbf{c}=\tilde{s} \mathbf{c}\right\} .
$$

Then $H_{\mathbf{c}, \tilde{s}}$ has codimension $g$ in $\mathfrak{H}_{g}$. Apply Condition ACZ to this $\tilde{s} \in \tilde{S}$ and $\mathbf{c} \in \mathbb{C}^{g}$. Hence we obtain a complex analytic variety $\tilde{C}$ of dimension $\operatorname{dim} S-g+1$ passing through $\tilde{s}$ such that $\tilde{C} \subset \tilde{S} \cap H_{\mathbf{c}, \tilde{s}}$. Now $\tilde{C} \subset H_{\mathbf{c}, \tilde{s}}$, so we have

$$
\operatorname{dim} \tilde{C}^{Z a r} \leq \operatorname{dim} H_{\mathbf{c}, \tilde{s}}=\operatorname{dim} \mathfrak{H}_{g}-g
$$

On the other hand $\tilde{C} \subset \tilde{S}$, so $u(\tilde{C}) \subset u(\tilde{S})=S$. Hence

$$
\operatorname{dim} u(\tilde{C})^{Z a r} \leq \operatorname{dim} S .
$$

Apply Ax-Schanuel, namely Theorem 10.2.5, to $\tilde{C}$. We obtain

$$
\operatorname{dim} \tilde{C}^{\mathrm{Zar}}+\operatorname{dim} u(\tilde{C})^{Z a r} \geq \operatorname{dim} \tilde{C}+\operatorname{dim} \tilde{C}^{\text {biZar }} .
$$

Assume $\tilde{C}^{\text {biZar }}=\mathfrak{H}_{g}$. Then we have $\left(\operatorname{dim} \mathfrak{H}_{g}-g\right)+\operatorname{dim} S \geq \operatorname{dim} \tilde{C}+\operatorname{dim} \mathfrak{H}_{g}$ by (10.1), (10.2) and (10.3). But this cannot hold since $\operatorname{dim} \tilde{C}=\operatorname{dim} S-g+1>0$. Hence $\tilde{C}^{b i Z a r} \neq \mathfrak{H}_{g}$. On the other hand $\operatorname{dim} \tilde{C}^{b i Z a r}>0$ since $\operatorname{dim} \tilde{C}=\operatorname{dim} S-g+1 \geq 1$. So we can take the desired $\tilde{F}$ to be $\tilde{C}^{b i Z a r}$.

Before moving on, we point out that we have not yet used the full strength of Condition ACZ since we did not vary the variable c. Now let us proof Theorem 10.1.1.

Proof of Theorem 10.1.1. Suppose Theorem 10.1.1 is not true. By condition (v) of Lemma 10.2.6, there exists a point $s \in S(\mathbb{C})$ such that $s$ is not contained in any proper bi-algebraic subvariety of $\mathcal{A}_{g}$ of positive dimension. Take $\tilde{s}$ to be a point in $u^{-1}(s)$ for this $s$. Applying Proposition 10.3.1 to $\tilde{s}$, we get a bi-algebraic subset $\tilde{F}$ of positive dimension, properly contained in $\mathfrak{H}_{g}$, such that $\tilde{s} \in \tilde{F}$. But then $u(\tilde{F})$ is a proper bi-algebraic subvariety of $\mathcal{A}_{g}$ of positive dimension which contains $s$. Now we get a contradition.

10.4. Proof of Theorem 10.1.3. In fact the same techniques for Theorem 10.1 .1 can be used to prove Theorem 10.1.3.

Proof of Theorem 10.1.3. Suppose we have an abelian scheme $A / S$ satisfying the three properties. Let $\mathbf{c} \in \mathbb{C}^{g}$ and $\tilde{s} \in \tilde{S}$ be as in condition (ii) of Theorem 10.1.3. Then for $H_{\mathbf{c}, \tilde{s}}=\left\{Z \in \mathfrak{H}_{g}: Z \mathbf{c}=\tilde{s} \mathbf{c}\right\} \subset \mathfrak{H}_{g}$, we have

$$
\operatorname{codim}_{\tilde{S} \text { biZar }}\left(H_{\mathbf{c}, \tilde{s}} \cap \tilde{S}^{\text {biZar }}\right)=g .
$$

Now that $\tilde{S}^{\text {biZar }}$ is affine linear in $\mathfrak{H}_{g}$ by Lemma 10.2.4. So for such a c, (10.4) holds for any $\tilde{s} \in \tilde{S}$ because $H_{\mathbf{c}, \tilde{s}}$ is also affine linear in $\mathfrak{H}_{g}$. Hence we may assume that $\tilde{s}$ is Hodge generic in $\tilde{S}$, namely $\operatorname{MT}(\tilde{s})=\operatorname{MT}(S)$.

Applying Condition ACZ to this $\tilde{s}$ and $\mathbf{c}$, we obtain a complex analytic variety $\tilde{C}$ of

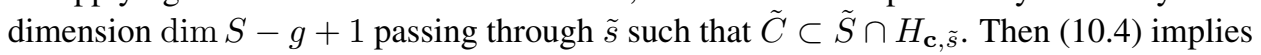

$$
\operatorname{dim} \tilde{C}^{Z a r} \leq \operatorname{dim}\left(H_{\mathbf{c}, \tilde{s}} \cap \tilde{S}^{\text {biZar }}\right)=\operatorname{dim} \tilde{S}^{\text {biZar }}-g .
$$

On the other hand $\tilde{C} \subset \tilde{S}$, so $u(\tilde{C}) \subset u(\tilde{S})=S$. Hence

$$
\operatorname{dim} u(\tilde{C})^{Z a r} \leq \operatorname{dim} S
$$


Apply Ax-Schanuel, namely Theorem 10.2.5, to $\tilde{C}$. We obtain (10.7) $\operatorname{dim} \tilde{C}^{Z a r}+\operatorname{dim} u(\tilde{C})^{Z a r} \geq \operatorname{dim} \tilde{C}+\operatorname{dim} \tilde{C}^{b i Z a r}=\operatorname{dim} S-g+1+\operatorname{dim} \tilde{C}^{\mathrm{biZar}}$.

By (10.5), (10.6) and (10.7), we get $\operatorname{dim} \tilde{S}^{\text {biZar }}>\operatorname{dim} \tilde{C}^{\text {biZar }}$. Thus in order to get a contradiction, it suffice to prove $\tilde{S}^{\text {biZar }}=\tilde{C}^{\text {biZar }}$. We shall use condition (i) of Theorem 10.1.3 to prove this fact.

The logarithmic Ax theorem for $\mathcal{A}_{g}$ says that $\tilde{S}^{\text {biZar }}=H_{S}^{\circ}(\mathbb{R})^{+} \tilde{s}$. We refer to [11, Theorem 8.1] for this theorem. Recall that $\tilde{s}$ is Hodge generic in $\tilde{S}$. Hence $H_{S}^{\circ}$ is normal in $\operatorname{MT}(\tilde{s})$ by André [2, Theorem 1].

By Ullmo-Yafaev [35, Theorem 1.2], bi-algebraic subsets of $\mathfrak{H}_{g}$ are precisely the weakly special subsets of $\mathfrak{H}_{g}$. Now $\tilde{C}^{\text {biZar }}$ contains $\tilde{s}$ which is Hodge generic in $\tilde{S}$, and $\tilde{C}^{\text {biZar }} \subset \tilde{S}^{\text {biZar }} \subset \operatorname{MT}(\tilde{s})(\mathbb{R})^{+} \tilde{s}$. So by definition of weakly special subvarieties (see [35, Definition 2.1] or [30, Definition 4.1.(b)]), we have $\tilde{C}^{\text {biZar }}=N(\mathbb{R})^{+} \tilde{s}$ for some normal subgroup $N$ of $\operatorname{MT}(\tilde{s})$. Now $N(\mathbb{R})^{+} \tilde{s} \subset H_{S}^{\circ}(\mathbb{R})^{+} \tilde{s}$, both $N$ and $H_{S}^{\circ}$ are normal subgroups of the reductive group $\mathrm{MT}(\tilde{s})$, and $H_{S}^{\circ}$ is simple by condition (i) of Theorem 10.1.3. Hence $N(\mathbb{R})^{+} \tilde{s}=H_{S}^{\circ}(\mathbb{R})^{+} \tilde{s}$, and so $\tilde{C}^{\text {biZar }}=\tilde{S}^{\text {biZar }}$.

Acknowledgements. We are pleased to thank Professor C. Voisin for sharing with us preliminary versions of her paper [36] and for interesting conversations with all of us. We thank Professors D. Bertrand, Ph. Griffiths, I. Krichever and B. Mazur for their interest in this work and helpful interactions. The third author thanks Professor J-P. Serre for several helpful comments, in particular pointing out inaccuracies in previous versions of section 9 and suggesting Theorem 9.1.1 in its present form. This research was partially supported by the grant DIMAGeometry PRIDZUCC of the University of Udine.

\section{REFERENCES}

[1] N. A'CAMPO - Tresses, monodromie et le groupe symplectique, Comment. Math. Helv. 54 (2) (1979), 318327.

[2] Y. ANDRÉ - Mumford-Tate groups of mixed Hodge structures and the theorem of the fixed part, Compos. Math. 82 (1992), 1-24.

[3] Y. ANDRÉ - Groupes de Galois motiviques et périodes, Séminaire Bourbaki, Novembre 201568 ème année, 2015-2016. S. F. M. Astérisque.

[4] Y. ANDRÉ - On the Kodaira-Spencer map of abelian schemes, Ann. Sc. Norm. Super. Pisa, Cl. Sci. (5) Vol. XVII (2017), 1397-1416.

[5] D. BERTRAND - Manin's theorem of the kernel : a remark on a paper of C-L. Chai, http://webusers.imjprg.fr/ daniel.bertrand/Recherche/rpdf/Manin_Chai.pdf

[6] M. BOROVOI - The Hodge group and endomorphism algebra of an Abelian variety, Problems in Group Theory and Homological Algebra, Yaroslav (1981), 124-126 (english version on the author's webpage).

[7] P. CORVAJA, D. MASSER, U. ZANNIER - Torsion Hypersurfaces on Abelian Schemes and Betti Coordinates, Math. Annalen, 371 (2018), 1013-1045.

[8] F. BEUKERS, W. BROWNAWELL, G. HECKMAN - Siegel normality, Annals of Math., 127(2) (1988), 279-308.

[9] P. DELIGNE - Théorie de Hodge III, Publ. Math. I.H.E.S. 44 (1974), 5-77.

[10] P. DELIGNE - Variétés de Shimura: interprétation modulaire, et techniques de construction de modèles canoniques, 247-289. In Automorphic forms, representations and L-functions (Corvallis 1977), Part 2, Proc. Sympos. Pure Math. XXXIII. A. M. S. Providence, R.I. (1979).

[11] Z. GAO - Towards the André-Oort conjecture for mixed Shimura varieties: the Ax-Lindemann-weierstrass theorem and lower bounds for Galois orbits of special points. J. Reine Angew. Math (Crelle), online.

[12] Z. GAO, P. HABEGGER - Heights in Families of Abelian Varieties and the Geometric Bogomolov Conjecture, preprint (2018), Annals of Math. 189 (2019), 527-604.

[13] Z. GAO - Generic rank of the Betti map and Unlikely Intersections, preprint (2018) https://arxiv.org/abs/1810.12929 
[14] Ph. GRIFFITHS, J. HARRIS - A Poncelet theorem in space, Commentarii Math. Helvetici 52 (1977), 145160.

[15] B. GROSS, J. HARRIS - Real Algebraic Curves, Ann. Sci. E. N. S. 14 (1981), 157-182.

[16] S. HELGASON - Differential Geometry, Lie Groups, and Symmetric Spaces, volume 34 of Graduate Studies in Mathematics. A. M. S. 2001.

[17] N. HITCHIN - Poncelet polygons and the Painlevé equations, Geometry and analysis (Bombay, 1992), 151-185, Tata Inst. Fund. Res., Bombay, 1995.

[18] N. KATZ - Algebraic solutions of differential equations (p-curvature and the Hodge filtration), Invent. Math. 18 (1972), 1-118.

[19] I. KRICHEVER - Nonlinear Equations and Elliptic Curves, Journal Soviet Math. 28 (1985), 51-90.

[20] B. LAWRENCE - A Density Result for Real Hyperelliptic Curves, Note C.R.A.S. Paris, 2016.

[21] S. LOJASIEWICZ - Introduction to complex analytic geometry, Springer, 1991.

[22] L. LOVÁSZ - Singular spaces of matrices and their application in combinatorics, Bol. Soc. Braz. Mat. 20 (1989), 87-99.

[23] Yu. MANIN - Rational points of algebraic curves over function fields Izv. Akad. Nauk SSSR 27 (1963), 1395-1440.

[24] N. MOK - Metric Rigidity Theorems on Hermitian Locally Symmetric Manifolds, volume 6 of Pure Mathematics. World Scientific, 1989.

[25] N. MOK, J. PILA, J. TSIMERMAN - Ax-Schanuel for Shimura varieties, Annals of Math. 189 (2019), 945-978.

[26] B. MOONEN - Linearity properties of Shimura varieties, I. Journal of Algebraic Geometry, 7(3) (1988) 539-567.

[27] S. MORI - The endomorphism rings of some abelian varieties, Japan J. Math 2, 1 (1976), 109-130.

[28] G. MUSTAFIN - Families of algebraic varieties and invariant cycles, Izv Akad Nauk SSSR 49 (1985), Math USSR Izvestia 27 (1986).

[29] G. OTTAVIANI - An introduction to the hyperdeterminant and to the rank of multidimensional matrices, in I. Peeva (ed.), Commutative Algebra, Dedicated to D. Eisenbud, 609-638, Springer, 2013.

[30] R. PINK - A combination of the conjectures of Mordell-Lang and André-Oort, In Geometric Methods in Algebra and Number Theory, volume 253 of Progress in Mathematics, pages 251-282. Birkhäuser, 2005.

[31] R. PINK - A Common Generalization of the Conjectures of André-Oort, Manin-Mumford, and MordellLang, https://people.math.ethz.ch/ pink/ftp/AOMMML.pdf (2005).

[32] R. ROBINSON - Conjugate algebraic integers in real point sets, Math. Zeitschrift 84 (1964), 415-427.

[33] J.-P. SERRE - Algèbre locale, multiplicités, LNM 11, Springer, 1965.

[34] J-P. SERRE - Distribution asymptotique des valeurs propres des endomorphismes de Frobenius (d'après Abel, Chebychev, Robinson...), Séminarie Bourbaki, Mars 2018.

[35] E. ULLMO, A. YAFAEV - A characterisation of special subvarieties, Mathematika, 57(2) (2011), 263-273.

[36] C. VOISIN - Torsion points of sections of Lagrangian torus fibrations and the Chow ring of hyper-Kähler fourfolds, Geometry of Moduli, Abel Symp. 14, Springer 2018.

[37] J. WILDESHAUS - The canonical construction of mixed sheaves on mixed Shimura varieties, in Realizations of Polylogarithms, Springer Lect. Notes in Math. 1650 (1997), 77-140.

[38] U. ZANNIER - Some Problems of Unlikely Intersections in Arithmetic and Geometry, Annals of Mathematics Studies 181, Princeton University Press, 2012.

Yves André, Institut Mathématique de Jussieu - Paris Rive Gauche, 4, Place Jussieu 75005 PARIS, FRANCE

Email address: yves. andred imj-prg. fr

Pietro Corvaja, Dipartimento di Scienze Matematiche, Informatiche e Fisiche, UniverSiTÀ Di Udine, Via DELlE SCIENZE, 206, Udine, ITALY

Email address: pietro.corvaja@uniud.it

Ziyang Gao, Institut Mathématique de Jussieu - Paris Rive gauche, 4, Place Jussieu 75005 PARIS, FRANCE

Email address: ziyang.gao@imj-prg.fr

Umberto Zannier, Scuola Normale Superiore, Piazza dei CaValieri, 7, 56126 Pisa, Italy

Email address: u. zannieresns. it 